TRANSACTIONS OF THE

AMERICAN MATHEMATICAL SOCIETY

Volume 350, Number 6, June 1998, Pages 2185-2227

S $0002-9947(98) 01937-0$

\title{
CARATHÉODORY-TOEPLITZ AND NEHARI PROBLEMS FOR MATRIX VALUED ALMOST PERIODIC FUNCTIONS
}

\author{
LEIBA RODMAN, ILYA M. SPITKOVSKY, AND HUGO J. WOERDEMAN
}

\begin{abstract}
In this paper the positive and strictly contractive extension problems for almost periodic matrix functions are treated. We present necessary and sufficient conditions for the existence of extensions in terms of Toeplitz and Hankel operators on Besicovitch spaces and Lebesgue spaces. Furthermore, when a solution exists a special extension (the band extension) is constructed which enjoys a maximum entropy property. A linear fractional parameterization of the set of all extensions is also provided. The techniques used in the proofs include factorizations of matrix valued almost periodic functions and a general algebraic scheme called the band method.
\end{abstract}

\section{INTRODUCTION}

We let $(A P)$ denote the algebra of complex valued almost periodic functions on the real line, i.e., the closed subalgebra of $L^{\infty}(\mathbb{R})$ generated by the functions $e^{i \lambda t}, \lambda \in \mathbb{R}$.

Recall that for any $f(t) \in(A P)$ the Fourier series is defined by the formal sum

$$
\sum_{\lambda} f_{\lambda} e^{i \lambda t}
$$

where

$$
f_{\lambda}=\lim _{T \rightarrow \infty} \frac{1}{2 T} \int_{-T}^{T} e^{-i \lambda t} f(t) d t, \quad \lambda \in \mathbb{R},
$$

and the sum in (1.1) is taken over the set $\sigma(f)=\left\{\lambda \in \mathbb{R}: f_{\lambda} \neq 0\right\}$, called the spectrum of $f(t)$. The spectrum of every $f \in(A P)$ is at most a countable set. The Wiener algebra $(A P W)$ is defined as the set of all $f(t) \in(A P)$ such that the Fourier series of $f(t)$ converges absolutely. For the general theory of almost periodic functions we refer the reader to the books $[31,32,6]$. In the next section, we present some basic properties of the algebras $(A P)$ and $(A P W)$.

Denote by $(A P W)^{m \times n}$ the set (algebra if $\left.m=n\right)$ of $m \times n$ matrices with entries in $(A P W)$. A matrix function $f \in(A P W)^{n \times n}$ is called positive if $f(t)$ is hermitian

Received by the editors April 29, 1996 and, in revised form, September 18, 1996.

1991 Mathematics Subject Classification. 42A75, 26A99, 15A54, 47A68, 47A56, 47A57, 42A82, 47B35.

Key words and phrases. Almost periodic matrix functions, contractive extensions, positive extensions, canonical factorization, Besicovitch space, Hankel operators, Toeplitz operators, band method.

The research is partially supported by NSF Grants 9500924 (LR, HJW) and 9401848 (IMS). The research of IMS was also supported by a semester research grant from the College of William \& Mary. 
for every $t \in \mathbb{R}$, and there exists an $\epsilon>0$ such that $f(t) \geq \epsilon I_{n}$ for all $t \in \mathbb{R}$, where $I_{n}$ is the $n \times n$ identity matrix. The inequality $X \geq Y$ between hermitian matrices $X$ and $Y$ means that $X-Y$ is positive semidefinite.

We say that $f \in(A P W)^{m \times n}$ is strictly contractive if there exists a $\delta<1$ such that

$$
\|f(t)\| \leq \delta \quad \text { for all } t \in \mathbb{R},
$$

where $\|A\|$ denotes the largest singular value of the matrix $A$.

In this paper we consider the Carathéodory-Toeplitz and Nehari problems for almost periodic matrix functions in the Wiener class. That is, we consider the following questions:

C-T: Let $\mu \in(0, \infty)$ and $f \in(A P W)^{n \times n}$ be such that $\sigma(f) \subseteq[-\mu, \mu]$. What is the condition on $f$ such that there exists a positive extension $h$ of $f$ ? In other words, when does there exist an $h \in(A P W)^{n \times n}$ such that

(1) $h$ is positive;

(2) $f_{\lambda}=h_{\lambda}, \lambda \in[-\mu, \mu]$ ?

In addition, if a positive extension exists, how do we construct one/all?

The $\mathbf{C}-\mathbf{T}$ problem is also known as the positive extension problem. In the scalar periodic case, namely, when $f$ and $h$ are scalar functions and $\sigma(f), \sigma(h) \subseteq \mathbb{Z}$, this is a classical problem which (in other formulations) goes back to $[5,39]$. The scalar almost periodic case has been treated before in [38].

$\mathbf{N}$ : Given is an $f \in(A P W)^{m \times n}$ such that $\sigma(f) \subseteq(-\infty, 0]$. When does there exist a strictly contractive extension $h \in(A P W)^{m \times n}$ of $f$, i.e., an $h$ such that

(1) $h$ is strictly contractive;

(2) $h_{\lambda}=f_{\lambda}, \lambda \leq 0$ ?

If such an $h$ exists, how do we construct one/all?

This problem is also known as a contractive extension problem. In the scalar periodic case ( $f$ and $h$ are scalar functions and $\sigma(f), \sigma(h) \subseteq \mathbb{Z}$ ) this is the classical Nehari problem (see [35]).

There is an extensive literature on the Carathéodory-Toeplitz and Nehari type problems, for various classes of matrix and operator functions (mainly for the periodic case), and their numerous applications, notably in signal processing and control theory. We mention here the books $[13,2,24,17]$, in which various aspects of positive and contractive extension problems and their applications are studied. Of particular relevance to the present paper are: the solutions of the periodic Nehari problem for matrix and scalar functions obtained in [1]; the maximum entropy principles which originated in [4] and have been developed in $[9,11]$; and the band method (including the maximum entropy principles) which was initiated in $[12,10]$ and further developed in [18]-[22], [40].

Often the Nehari problem appears as the distance problem:

Distance problem. Given $f \in(A P W)^{m \times n}$, find the distance from $f$ to the set $(A P W)_{(0, \infty)}^{m \times n}$ of all functions in $(A P W)^{m \times n}$ whose spectrum lies in $(0, \infty)$. In 
other words, find

$$
\Delta(f)=\inf _{g \in(A P W)_{(0, \infty)}^{m \times n}} \sup _{t \in \mathbb{R}}\|f(t)+g(t)\|
$$

Moreover, for every $\Delta>\Delta(f)$ find all $g \in(A P W)_{(0, \infty)}^{m \times n}$ such that

$$
\sup _{t \in \mathbb{R}}\|f(t)+g(t)\|<\Delta \text {. }
$$

The solution to the distance problem can be obtained by a scaling of the function $f(t)$ (replacing $f(t)$ by $k f(t)$, where $k$ is a suitable constant), and using the solution of the corresponding Nehari problem.

We obtain complete solutions of the Carathéodory-Toeplitz and Nehari problems as formulated above. Moreover, we prove more detailed results relating the spectrum of $f(t)$ to the spectrum of its possible positive (or strictly contractive) extensions $h(t)$. For example, if such an extension exists at all, then an extension $h(t)$ exists having its spectrum $\sigma(h)$ in the set of all finite linear combinations with integer coefficients of the numbers in $\sigma(f)$. This allows us to consider simultaneously many special classes of matrix valued almost periodic functions, for example, the periodic functions. Our main results are stated in Section 3 and 4.

For both $\mathbf{C}-\mathbf{T}$ and $\mathbf{N}$ problems we shall employ the band method, a general algebraic scheme for solving positive and contractive extension problems. The references [20] and Chapter XXXIV in [17] both contain a detailed exposition of the band method. Other ingredients needed in the proofs of our main theorems include certain properties of Riemann and Hankel operators on the Besicovitch space (Section 5), factorization results (Section 6) and results on invertibility of elements in Banach algebras (Section 7). Most of the material in Section 5 is borrowed from [27]. We would like to thank Yu. Karlovich for several useful presentations and discussions concerning these results.

The proofs of our main results are given in Sections 8 and 9. In Section 10 we consider variations of the $\mathbf{C}-\mathbf{T}$ and $\mathbf{N}$ problems in which $f_{ \pm \mu}$ is not given (in the $\mathbf{C}-\mathbf{T}$ problem) or $f_{0}$ is not given (in the $\mathbf{N}$ problem). It turns out that these "point excluding" variations can be studied in much the same manner as the $\mathbf{C}-\mathbf{T}$ and $\mathbf{N}$ problems above. Finally, in Section 11 a joint $H^{\infty} / H^{2}$ bound is given for the solutions of the Nehari problem.

We use the following notations and conventions throughout the paper. $\mathbb{R}, \mathbb{C}, \mathbb{Z}$ stand for the real numbers, complex numbers, and integers, respectively. The Banach spaces used here are: $(A P) ;(A P W) ;(B)$, the Besicovitch space (see Section 3 for the definition); $\left(H^{2 \pm}\right)$ and $\left(H^{\infty \pm}\right)$, the Hardy spaces of analytic functions on the upper halfplane (with the sign + ) or the lower halfplane (with the sign - ); $\left(L^{p}\right)$ and $\left(L^{\infty}\right)$, Lebesgue spaces. If $(X)$ is a set (typically a Banach space or an algebra), we denote by $(X)^{m \times n}$ the set of $m \times n$ matrices with entries in $(X)$. All operators are assumed to be linear and bounded. Scalar products in various Hilbert spaces are denoted by $\langle\cdot, \cdot\rangle$.

\section{Preliminaries on Almost Periodic Functions}

We review here some basic facts about almost periodic functions. Many of these facts with full proofs can be found in $[31,32,6]$. 
A continuous complex valued function $f(t), t \in \mathbb{R}$, is called almost periodic if for every $\epsilon>0$ there exists $L>0$ such that every interval of length $L$ contains at least one point $\tau \in \mathbb{R}$ for which

$$
|f(t+\tau)-f(t)|<\epsilon \quad \text { for all } t \in \mathbb{R} .
$$

(This definition is equivalent to the definition given in the introduction.) Denote by $(A P)$ the class of almost periodic functions. Every almost periodic function is bounded; define $\|f\|=\sup _{t \in \mathbb{R}}|f(t)|, f \in(A P)$.

Proposition 2.1. (a) $(A P)$ is a closed subalgebra in $L^{\infty}(\mathbb{R})$; moreover, if $f \in$ $(A P)$, then $|f|, f^{*} \in(A P)$, where $f^{*}$ is defined by $f^{*}(t)=\overline{f(t)}, t \in \mathbb{R}$; if $f \in(A P)$ and $\inf _{T \in \mathbb{R}}|f(t)|>0$, then $\frac{1}{f} \in(A P)$.

(b) For every $f \in(A P)$ there exists

$$
\lim _{T \rightarrow \infty} \frac{1}{2 T} \int_{-T}^{T} f(t) d t
$$

called the mean of $f$, and denoted $M\{f\}$.

Using Proposition 2.1 (b), for $f \in(A P)$ we define

$$
f_{\lambda}=M\left\{e^{-i \lambda t} f(t)\right\}, \lambda \in \mathbb{R} .
$$

The set $\sigma(f)$ of all $\lambda \in \mathbb{R}$ for which $f_{\lambda} \neq 0$ is called the spectrum of $f$. The spectrum is at most a countable set. We associate with $f$ the (formal) Fourier series

$$
f(t) \sim \sum_{\lambda \in \sigma(f)} f_{\lambda} e^{i \lambda t}
$$

We have the multiplication formula

$$
M\left\{f(t) g(t) e^{-\lambda t}\right\}=\sum_{\mu+\nu=\lambda} f_{\mu} g_{\nu}, \quad \lambda \in \mathbb{R}
$$

where $f, g \in(A P)$, and the uniqueness theorem: if $f, g \in(A P)$ have the same Fourier series, then $f(t)=g(t), t \in \mathbb{R}$.

An almost periodic function $f$ is said to belong to the Wiener class if $\sum_{\lambda \in \sigma(f)}\left|f_{\lambda}\right|<$ $\infty$. In this case, $f$ coincides with the sum of its Fourier series:

$$
f(t)=\sum_{\lambda \in \sigma(f)} f_{\lambda} e^{i \lambda t}, \quad t \in \mathbb{R} .
$$

Denote by $(A P W)$ the Wiener class of almost periodic functions.

Proposition 2.2. (a) $(A P W)$ is a Banach $*$-algebra (with respect to the pointwise addition and multiplication) with the norm

$$
\|f\|_{W}=\sum_{\lambda \in \sigma(f)}\left|f_{\lambda}\right| .
$$

Note that $\|f\| \leq\|f\|_{W}$.

(b) If $f \in(A P W)$, then $\frac{1}{f} \in(A P W)$ if and only if $\inf _{t \in \mathbb{R}}|f(t)|>0$.

(c) Let $f \in(A P W)$, and let $f(\mathbb{R})=\{f(t): t \in \mathbb{R}\}$ be the set of values of $f$. If $F(z)$ is an analytic function defined in an open neighborhood of the closure of $f(\mathbb{R})$, then $F(f(t)) \in(A P W)$. 
Part (c) is Corollary 2, p. 175 in [16], whereas part (b) (which is actually a particular case of part (c)) is Corollary 1, p. 175 in [16].

For matrix functions, Proposition 2.2 (c) leads to the next result. We first observe that a matrix function $f \in(A P W)^{n \times n}$ is invertible in $(A P W)^{n \times n}$ if and only if

$$
\inf _{t \in \mathbb{R}}|\operatorname{det} f(t)|>0 .
$$

Indeed, if (2.2) holds, then $\frac{1}{\operatorname{det} f(t)} \in(A P W)$ by Proposition $2.2(\mathrm{~b})$, and therefore

$$
f^{-1}=\frac{\operatorname{adjoint}(f)}{\operatorname{det} f} \in(A P W)^{n \times n} .
$$

Conversely, if $f^{-1} \in(A P W)^{n \times n}$, then clearly det $f(t) \neq 0$ for all $t \in \mathbb{R}$. Since $f^{-1}$ is uniformly bounded on the real line, the eigenvalues of $f^{-1}$ are also uniformly bounded. Hence the eigenvalues of $f$ are uniformly bounded away from zero, and (2.2) holds.

Proposition 2.3. Let $f \in(A P W)^{n \times n}$, and let

$$
\Omega=\{z \in \mathbb{C}: z \text { is an eigenvalue of } f(t) \text { for some } t \in \mathbb{R}\} .
$$

If $\Psi(z)$ is an analytic function in an open neighborhood of the closure of $\Omega$, then $\Psi(f(t)) \in(A P W)^{n \times n}$.

Here, for every fixed $t \in \mathbb{R}, \Psi(f(t))$ is understood as the $n \times n$ matrix defined by the standard functional calculus.

Proof. Since $f(t)$ is a bounded function, the set $\Omega$ is also bounded. Let $z_{0} \notin \bar{\Omega}$ (the closure of $\Omega$ ). Then $z_{0} I-f(t)$ has eigenvalues $z_{0}-\lambda_{1}(t), \ldots, z_{0}-\lambda_{n}(t)$, where $\lambda_{1}(t), \ldots, \lambda_{n}(t)$ are eigenvalues of $f(t)$, so

$$
\left|\operatorname{det}\left(z_{0} I-f(t)\right)\right|=\prod_{j=1}^{n}\left|z_{0}-\lambda_{j}(t)\right| \geq \alpha^{n}
$$

where $\alpha$ is the (positive) distance from $z_{0}$ to $\bar{\Omega}$. By the observation (2.2) the matrix function $z_{0} I-f$ is invertible in $(A P W)^{n \times n}$. Thus, $z_{0}$ belongs to the resolvent set of $f$ as an element of the Banach algebra $(A P W)^{n \times n}$. Now we can define $\Psi(f) \in(A P W)^{n \times n}$ using the functional calculus:

$$
\Psi(f)=\frac{1}{2 \pi i} \int_{\Gamma} \Psi(z)(z I-f)^{-1} d z
$$

for a suitable contour $\Gamma$, where the integral converges in the norm of $(A P W)^{n \times n}$. Since the convergence in $(A P W)^{n \times n}$ implies pointwise convergence, for every $t \in \mathbb{R}$ we have

$$
(\Psi(f))(t)=\frac{1}{2 \pi i} \int_{\Gamma} \Psi(z)(z I-f(t))^{-1} d z .
$$

But the right-hand side of (2.3) is just the definition of $\Psi(f(t))$. It follows that $\Psi(f(t))$ (defined pointwise for every $t \in \mathbb{R}$ ) is the value of $\Psi(f)$ at $t$; since $\Psi(f) \in$ $(A P W)^{n \times n}$, we are done.

We consider next the sets of almost periodic functions and Wiener class functions, which are defined by the spectrum location. Let $\Delta$ be a non-empty subset of the 
real line. Denote

$$
\begin{aligned}
(A P)_{\Delta} & =\{f \in(A P): \sigma(f) \subseteq \Delta\} \\
(A P W)_{\Delta} & =\{f \in(A P W): \sigma(f) \subseteq \Delta\} .
\end{aligned}
$$

Proposition 2.4. (a) $(A P)_{\Delta}$ is a closed subspace in the Banach algebra $(A P)$ and $(A P W)_{\Delta}$ is a closed subspace in the Banach algebra $(A P W)$.

(b) If $\Delta$ is an additive semigroup (i.e., $\alpha, \beta \in \Delta \Rightarrow \alpha+\beta \in \Delta$ ), then $(A P)_{\Delta}$ (resp. $\left.(A P W)_{\Delta}\right)$ is a subalgebra in $(A P)$ (resp. $\left.(A P W)\right)$.

(c) If $\Delta$ is an additive semigroup such that $0 \in \Delta$, and $\{\alpha+\beta=0$ for $\alpha, \beta \in$ $\Delta \Rightarrow \alpha=\beta=0\}$, then the mean is multiplicative on $(A P)_{\Delta}$ :

$$
M\{f g\}=M\{f\} \cdot M\{g\} \quad \text { for every } f, g \in(A P)_{\Delta} .
$$

Proof. Since for every $\lambda \in \mathbb{R}, f \mapsto f_{\lambda}$ is a linear functional on $(A P)$ and on $(A P W)$, the sets $(A P)_{\Delta}$ and $(A P W)_{\Delta}$ are clearly linear sets. The inequality

$$
\left|M\left\{e^{-i \lambda t} f(t)\right\}-M\left\{e^{-i \lambda t} g(t)\right\}\right| \leq\|f-g\|, \quad f, g \in(A P),
$$

shows that $(A P)_{\Delta}$ and $(A P W)_{\Delta}$ are closed in $(A P)$ and $(A P W)$, respectively. Finally, (2.1) proves (b) and (c) if we take into account that $M\{f\}=f_{0}$.

We will also need results asserting that compositions of almost periodic functions in $(A P)_{\Delta}$ are again in the same class $(A P)_{\Delta}$ (under certain hypotheses). A very general result along these lines can be stated as follows:

Proposition 2.5. Let $\Sigma \subseteq \mathbb{R}$ be an additive subgroup (in other words, $\Sigma \neq \emptyset$ and $x, y \in \Sigma \Rightarrow x-y \in \bar{\Sigma})$. Let $G\left(z_{1}, \ldots, z_{n}\right)$ be a uniformly continuous function of $\left(z_{1}, \ldots, t_{n}\right) \in \mathcal{M}$, where $\mathcal{M} \subseteq \mathbb{C}^{n}$. If $f_{1}, \ldots, f_{n} \in(A P)_{\Sigma}$ are such that $\left(f_{1}(t), \ldots, f_{n}(t)\right) \in \mathcal{M}$ for every $t \in \mathbb{R}$, then $G\left(f_{1}(t), \ldots, f_{n}(t)\right) \in(A P)_{\Sigma}$.

The proof follows from Theorem 1.7 in [6] and its proof.

Corollary 2.6. Let $\Sigma \subseteq \mathbb{R}$ be an additive subgroup. If $f \in(A P W)_{\Sigma}$ is real valued and $\inf _{t \in \mathbb{R}} f(t)>0$, then $\log f \in(A P W)_{\Sigma}$.

To prove this corollary, combine Propositions 2.2 (c) and 2.5.

For a matrix valued function $f \in(A P)^{m \times n}$ we define the $m \times n$ matrix $M\{f\}$ by taking the means of the entries of $f$. The Fourier series $\sum_{\lambda \in \sigma(f)} f_{\lambda} e^{i \lambda t}$ is also defined entrywise, with

$$
\sigma(f)=\left\{\lambda \in \mathbb{R}: f_{\lambda} \neq 0\right\}
$$

as in the scalar case.

Corollary 2.7. Let $F \in(A P)^{n \times n}$ be invertible in $(A P)^{n \times n}$ and such that $\sigma(F) \subseteq$ $\Sigma$, where $\Sigma \subseteq \mathbb{R}$ is an additive subgroup. Then $\sigma\left(F^{-1}\right) \subseteq \Sigma$.

Proof. Using the determinants, we reduce the proof to the scalar case. In the scalar case, use Proposition 2.5 with $\mathcal{M}=\{z \in \mathbb{C}:|z| \geq \epsilon\}$ and $G(z)=z^{-1}$ (for a suitable $\epsilon>0)$.

We note that Corollaries 2.6 and 2.7 can also be proved using the following result (Theorem 2.7.1 in [31]).

Proposition 2.8. Let $\Sigma$ be an additive subgroup in $\mathbb{R}$. Let $F \in(A P)_{\Sigma}$ be such that $\inf _{t \in \mathbb{R}}|F(t)|>0$. Then $F(t)=e^{i c t} \cdot e^{f(t)}$, where $c \in \Sigma$ is a constant and $f \in(A P)_{\Sigma}$. 
Finally, we need a result on existence of the logarithm of determinants with a given location of the spectrum.

Proposition 2.9. Let $\Sigma \subseteq \mathbb{R}$ be an additive subgroup, and let there be given $F \in$ $(A P)_{[0, \infty) \cap \Sigma}^{n \times n}$ (resp. $\left.F \in(A P W)_{[0, \infty) \cap \Sigma}^{n \times n}\right)$ such that $\inf _{t \in \mathbb{R}}|\operatorname{det} F(t)|>0$ and $M\{F\}=$

I. Assume, in addition, that $F^{-1} \in(A P)_{[0, \infty)}^{n \times n}$. Then there is a branch $f$ of $\log (\operatorname{det} F)$ which is continuous on $\mathbb{R}$ and such that $f \in(A P)_{[0, \infty) \cap \Sigma}$ (resp. $f \in$ $(A P)_{[0, \infty) \cap \Sigma}^{n \times n}$ and $M\{f\}=0$.

Proof. We will prove the proposition for the case when $F \in(A P)_{[0, \infty) \cap \Sigma}^{n \times n}$ (the proof for the case when $F \in(A P W)_{[0, \infty) \cap \Sigma}^{n \times n}$ is analogous).

Observe first of all that $F^{-1} \in(A P)_{[0, \infty) \cap \Sigma}^{n \times n}$ by Corollary 2.7. Furthermore, $(\operatorname{det} F)^{ \pm 1} \in(A P)_{[0, \infty) \cap \Sigma}$, and by the multiplicativity properties of the mean (Proposition $2.4(\mathrm{c})$ ) $M\{\operatorname{det} F\}=\operatorname{det} M\{F\}=1$. Therefore, it suffices to consider the case of a scalar function $F$.

Denote by $\Theta$ the set of all scalar functions $F$ for which the statement of Proposition 2.9 holds. In other words,

$$
\Theta=\left\{F=e^{g}: g \in(A P)_{[0, \infty) \cap \Sigma}, M\{g\}=0\right\} .
$$

This set has the following properties:

(1) $\Theta$ is a multiplicative group.

Indeed, if $e^{g_{1}} \in \Theta, e^{g_{2}} \in \Theta$, then $e^{g_{1}} \cdot e^{g_{2}}=e^{g_{1}+g_{2}} \in \Theta$.

(2) If $\chi \in(A P)_{(0, \infty) \cap \Sigma},\|\chi\|<1$, then $1+\chi \in \Theta$.

To prove (2), one may choose

$$
g=\sum_{n=1}^{\infty} \frac{(-1)^{n+1}}{n} \chi^{n} .
$$

It follows from (1) and (2) that

(3) $\Theta$ is an open subset of $\left\{F \in(A P)_{[0, \infty) \cap \Sigma}: M\{F\}=1\right\}$.

At the same time,

(4) $\Theta$ is a closed subset of

$$
X=\left\{F \in(A P)_{[0, \infty) \cap \Sigma}: F^{-1} \in(A P)_{[0, \infty)} ; M\{F\}=1\right\} .
$$

Indeed, if $F_{n} \rightarrow F$ and $F^{ \pm 1} \in(A P)_{[0, \infty) \cap \Sigma}$, then $F_{n}^{-1} \rightarrow F^{-1}$ in the norm of $(A P)$, so that in the representation $F=F_{n}\left(1+F_{n}^{-1}\left(F-F_{n}\right)\right)$ the second multiple $1+F_{n}^{-1}\left(F-F_{n}\right)$ satisfies (2) for $n$ large enough.

We now prove that the set $X$ is connected. Using small (in the norm of $(A P)$ ) perturbations, we may restrict ourselves to the case of $(A P)$-polynomials, i.e., functions with finite spectrum. In this case, if $F \in X$ is an $(A P)$-polynomial, then

$$
F_{\alpha}(t)=F\left(t+i \frac{\alpha}{1-\alpha}\right), \quad \alpha \in[0,1],
$$

is a homotopy connecting $F_{0}=F$ with $F_{1}=M\{F\}=1$ without leaving $X$.

Being an open, closed, and non-empty subset of $X, \Theta$ has to coincide with $X$.

Observe that the additional hypotheses that $\sigma\left(F^{-1}\right) \subseteq[0, \infty)$ is essential in Proposition 2.9 . 


\section{Positive Extension Problem: The Main Theorems}

Our main results in this and the next section will be stated in terms of operators on the vector-valued Besicovitch space. We recall here the definition of this space (more information is found in Section 5). Introduce a scalar product on $(A P)$ by the formula

$$
\langle f, g\rangle=M\left\{f g^{*}\right\}, \quad f, g \in(A P) .
$$

The completion of $(A P)$ with respect to this scalar product is called the Besicovitch space and is denoted $(B)$. Thus $(B)$ is a Hilbert space.

For a nonempty set $\Lambda \subseteq \mathbb{R}$, define the projection

$$
\Pi_{\Lambda}\left(\sum_{\lambda \in \sigma(f)} f_{\lambda} e^{i \lambda t}\right)=\sum_{\lambda \in \sigma(f) \cap \Lambda} f_{\lambda} e^{i \lambda t},
$$

where $f \in(A P W)$. The projection $\Pi_{\Lambda}$ extends by continuity to the orthogonal projection (also denoted $\Pi_{\Lambda}$ ) on $(B)$. We denote by $(B)_{\Lambda}$ the range of $\Pi_{\Lambda}$, or, equivalently, the completion of $(A P)_{\Lambda}$ with the respect to the scalar product (3.1). The vector valued Besicovitch space $(B)^{n \times 1}$ consists of $n \times 1$ columns with components in $(B)$, with the standard Hilbert space structure. Similarly, $(B)_{\Lambda}^{n \times 1}$ is the Hilbert space of $n \times 1$ columns with components in $(B)_{\Lambda}$.

Recall that a Hilbert space operator $\mathbf{T}: \mathcal{H} \rightarrow \mathcal{H}$ is called positive definite (notation: $T>0$ ) if there exists an $\epsilon>0$ such that

$$
\langle\mathbf{T} f, f\rangle \geq \epsilon\|f\|^{2} \quad \text { for all } f \in \mathcal{H} .
$$

Consider first the $\mathbf{C}-\mathbf{T}$ problem (positive extension). In the following theorem and elsewhere in the paper, if $x=\left[x_{i j}(t)\right]_{i=1, j=1}^{m, n} \in(A P)^{m \times n}$, we denote the adjoint by $x^{*}=\left[y_{j k}(t)\right]_{j=1, k=1}^{n, m} \in(A P)^{n \times m}$, where $y_{j k}(t)=\overline{x_{k j}(t)}$; for a square invertible $x \in(A P)^{n \times n}$ we write $x^{*-1}$ for $\left(x^{*}\right)^{-1}=\left(x^{-1}\right)^{*}$.

Theorem 3.1. Let $\Sigma \subseteq \mathbb{R}$ be an additive subgroup, and let $\mu \in \Sigma, \mu>0$, be given. For a given function $f \in(A P W)_{[-\mu, \mu] \cap \Sigma}^{n \times n}$ the following statements are equivalent:

(i) $f$ has a positive extension in $(A P W)_{\Sigma}^{n \times n}$, i.e., there exists $h \in(A P W)_{\Sigma}^{n \times n}$ such that $h_{\lambda}=f_{\lambda}$ for all $\lambda \in[-\mu, \mu] \cap \Sigma$ and there is an $\epsilon>0$ such that $h(x) \geq \epsilon I$ for all $x \in \mathbb{R}$;

(ii) the generalized Toeplitz operator

$$
\mathbf{T}(f)_{[0, \mu] \cap \Sigma}:(B)_{[0, \mu] \cap \Sigma}^{n \times 1} \rightarrow(B)_{[0, \mu] \cap \Sigma}^{n \times 1},
$$

$$
g \mapsto \Pi_{[0, \mu]}(f g), g \in(B)_{[0, \mu] \cap \Sigma}^{n \times 1},
$$

is positive definite;

(iii) the generalized Toeplitz operator

$$
\mathbf{T}(f)_{[-\mu, 0] \cap \Sigma}:(B)_{[-\mu, 0] \cap \Sigma}^{n \times 1} \rightarrow(B)_{[-\mu, 0] \cap \Sigma}^{n \times 1}
$$

defined by $g \mapsto \Pi_{[-\mu, 0]}(f g)$ is positive definite.

(iv) $f$ has a positive extension in $(A P W)^{n \times n}$; 
(v) the generalized Toeplitz operator

$$
\mathbf{T}(f)_{[0, \mu]}:(B)_{[0, \mu]}^{n \times 1} \rightarrow(B)_{[0, \mu]}^{n \times 1}
$$

defined by (3.2) (where $g \in(B)_{[0, \mu]}^{n \times 1}$ ) is positive definite;

(vi) the generalized Toeplitz operator

$$
\mathbf{T}(f)_{[-\mu, 0]}:(B)_{[-\mu, 0]}^{n \times 1} \rightarrow(B)_{[-\mu, 0]}^{n \times 1}
$$

is positive definite.

When one (and thus all) of (i)-(vi) is satisfied, then

$$
h_{0}(t)=x^{*-1}(t) M\{x\} x^{-1}(t)=y^{*-1}(t) M\{y\} y^{-1}(t), \quad t \in \mathbb{R},
$$

is a positive extension of $f$ in $(A P W)_{\Sigma}^{n \times n}$. Here $x=\left(x_{i j}\right)_{i, j=1}^{n} \in(A P W)_{[0, \mu] \cap \Sigma}^{n \times n}$ and $y=\left(y_{i j}\right)_{i, j=1}^{n} \in(A P W)_{[-\mu, 0] \cap \Sigma}^{n \times n}$ are given via

$$
\left(x_{i j}\right)_{i=1}^{n}=\left(\mathbf{T}(f)_{[0, \mu] \cap \Sigma}\right)^{-1}\left(e_{j}\right) ;\left(y_{i j}\right)_{i=1}^{n}=\left(\mathbf{T}(f)_{[-\mu, 0] \cap \Sigma}\right)^{-1}\left(e_{j}\right),
$$

where $e_{j}$ denotes the $j^{\text {th }}$ column of the constant, almost periodic matrix function whose value at each point is the $n \times n$ identity matrix.

Of particular interest in Theorem 3.1, as well as in Theorems 3.2 and 3.3 below, are the cases when $\Sigma=\mathbb{R}$ and when $\Sigma=\mathbb{Z}$. The former case corresponds to the Carathéodory-Toeplitz problem stated in the introduction, while the latter case corresponds to the Carathéodory-Toeplitz problem in the Wiener algebra on the unit circle (see [9, 18]; also [33, 34]).

Note that formula (3.3) implies that we are required to show that $x$ and $y$ are invertible in $(A P W)_{\Sigma}^{n \times n}$, and that $M\{x\}$ and $M\{y\}$ are positive definite matrices. In addition, we shall show that

$$
x^{-1} \in(A P W)_{[0, \infty) \cap \Sigma}^{n \times n}, \quad y^{-1} \in(A P W)_{(-\infty, 0] \cap \Sigma}^{n \times n},
$$

so that (3.3) actually provides the spectral factorizations (see Section 6) of the positive extension $h_{0}(t)$. Furthermore, observe that

$$
\sigma\left(h_{0}^{-1}\right) \subseteq[-\mu, \mu] .
$$

Actually, we shall show that $h_{0}$ is the only positive extension with property (3.5) and in addition has a "maximum entropy" property. Before we can state the precise result we need to introduce the entropy. Let $f \in(A P W)^{n \times n}$ be positive definite. From Proposition $2.2(\mathrm{c})$, it follows that $\log (\operatorname{det} f)$ belongs to $(A P W)$. The number

$$
\Delta(f):=M\{\log (\operatorname{det} f)\}
$$

will be referred to as the entropy of $f$.

Theorem 3.2. Let $\mu$ and $\Sigma$ be as in Theorem 3.1. Let $f \in(A P W)_{[-\mu, \mu] \cap \Sigma}^{n \times n}$ be such that $\mathbf{T}(f)_{[0, \mu] \cap \Sigma}>0$ (or equivalently, $\mathbf{T}(f)_{[-\mu, 0] \cap \Sigma}>0$ ).

Let $h_{0}$ be defined by (3.3). Then $h_{0}$ is the unique positive extension in $(A P W)^{n \times n}$ of $f$ with property (3.5). Moreover, if $h$ is a positive extension in $(A P W)^{n \times n}$ of $f$, then

$$
\Delta\left(h_{0}\right) \geq \Delta(h),
$$

with equality if and only if $h_{0}=h$. 
In fact, we shall prove a stronger result, stating that if $D(h)$ is the constant (necessarily positive definite) matrix in the factorization $h=h_{+}^{*} D(h) h_{+}$, where $\left(h_{+}\right)^{ \pm 1} \in(A P W)_{[0, \infty)}^{n \times n}$ and $M\left\{h_{+}\right\}=I$, then $D\left(h_{0}\right) \geq D(h)$ for every positive extension of $f$, with the equality $D\left(h_{0}\right)=D(h)$ only if $h=h_{0}$. Using subsequently the observation that $\Delta(h)=\log (\operatorname{det} D(h))$, one may derive Theorem 3.2 from the latter result.

The third main result in this section concerns a description of all positive extensions in $(A P W)_{\Sigma}^{n \times n}$ using the parameter set

$$
(C A P W)_{(\mu, \infty) \cap \Sigma}^{n \times n}:=\left\{g \in(A P W)_{(\mu, \infty) \cap \Sigma}^{n \times n}: \sup _{t \in \mathbb{R}}\|g(t)\|<1\right\} .
$$

We denote by $\|A\|$ the operator norm (= the largest singular value) of a matrix $A$.

Theorem 3.3. Let $f, x$, and $y$ be as in Theorem 3.1, and let

$$
u=x M\{x\}^{-\frac{1}{2}}, \quad v=y M\{y\}^{-\frac{1}{2}} .
$$

Then each positive extension in $(A P W)_{\Sigma}^{n \times n}$ of $f$ is of the form

$$
T(g)=(u+v g)^{*-1}\left(I-g^{*} g\right)(u+v g)^{-1},
$$

where $g \in(C A P W)_{(\mu, \infty) \cap \Sigma}^{n \times n}$. Also, the correspondence between $(C A P W)_{(\mu, \infty) \cap \Sigma}^{n \times n}$ and the set of positive extensions in $(A P W)_{\Sigma}^{n \times n}$ of $f$ is one-to-one.

The proofs of Theorems 3.1, 3.2, and 3.3 will be given in Section 8 .

\section{Contractive Extension Problem: The Main Theorems}

Recall the almost periodic Nehari problem stated in the introduction: Given $f \in(A P W)^{m \times n}$, find if possible $\widetilde{f} \in(A P W)_{(0, \infty)}^{m \times n}$ such that

$$
\sup _{t \in \mathbb{R}}\|f(t)+\widetilde{f}(t)\|<1 .
$$

As for the Carathéodory-Toeplitz problem (see Section 3), we state and prove our results in a more general framework that takes into account the location of the spectra of $f$ and of $\tilde{f}$ in a given additive subgroup.

In the spirit of the classical Nehari problem (the periodic case, see [35]), we shall see that the necessary and sufficient conditions for existence of solutions $\widetilde{f}$ as above are given in terms of Hankel operators.

Let $\Sigma \subseteq \mathbb{R}$ be an additive subgroup, and let $f \in(A P W)_{(-\infty, 0] \cap \Sigma}^{m \times n}$. We introduce the following generalized Hankel operator:

$$
\mathbf{H}(f)_{\Sigma}:(B)_{[0, \infty) \cap \Sigma}^{n \times 1} \rightarrow(B)_{(-\infty, 0] \cap \Sigma}^{m \times 1}
$$

by

$$
\mathbf{H}(f)_{\Sigma} g=\Pi_{(-\infty, 0]}(f g), \quad g \in(B)_{[0, \infty) \cap \Sigma}^{n \times 1} .
$$

Note that, since $\Sigma$ is an additive subgroup, $\sigma(f g) \subseteq \Sigma$ for every $g \in(B)_{[0, \infty) \cap \Sigma}^{n \times 1}$ (see Proposition $2.4(\mathrm{~b})$ ), and therefore, indeed, $\mathbf{H}(f)_{\Sigma} g \in(B)_{(-\infty, 0] \cap \Sigma}^{m \times 1}$.

Our main results are the following.

Theorem 4.1. Let $f \in(A P W)_{(-\infty, 0] \cap \Sigma}^{m \times n}$ be given, where $\Sigma \subseteq \mathbb{R}$ is an additive subgroup. The following statements are equivalent: 
(i) $f$ has a strictly contractive extension in $(A P W)^{m \times n}$, i.e., there exists $g \in$ $(A P W)^{m \times n}$ such that $g_{\lambda}=f_{\lambda}$ for all $\lambda \leq 0$ and $\sup _{t \in \mathbb{R}}\|g(t)\|<1$;

(ii) the generalized Hankel operator

$$
\mathbf{H}(f):(B)_{[0, \infty)}^{n \times 1} \rightarrow(B)_{(-\infty, 0]}^{m \times 1}
$$

defined by (4.1) with $\Sigma=\mathbb{R}$ is a strict contraction (i.e., $\|\mathbf{H}(f)\|<1$ );

(iii) $f$ has a strictly contractive extension in $(A P W)_{\Sigma}^{m \times n}$, i.e., there exists $g \in$ $(A P W)_{\Sigma}^{m \times n}$ such that $g_{\lambda}=f_{\lambda}$ for all $\lambda \leq 0$ and $\sup _{g \in \mathbb{R}}\|g(t)\|<1$;

(iv) the generalized Hankel operator $\mathbf{H}(f)_{\Sigma}$ is a strict contraction.

When one (and thus all) of (i)-(iv) is satisfied, put

$$
\begin{aligned}
\widehat{\alpha}(t) & =\left[I-\mathbf{H}(f)_{\Sigma}\left(\mathbf{H}(f)_{\Sigma}\right)^{*}\right]^{-1}\left(I_{m}\right), \\
\widehat{\beta}(t) & =\mathbf{H}(f)_{\Sigma}\left[I-\left(\mathbf{H}(f)_{\Sigma}\right)^{*} \mathbf{H}(f)_{\Sigma}\right]^{-1}\left(I_{n}\right), \\
\widehat{\gamma}(t) & =\left(\mathbf{H}(f)_{\Sigma}\right)^{*}\left[I-\left(\mathbf{H}(f)_{\Sigma}\left(\mathbf{H}(f)_{\Sigma}\right)^{*}\right]^{-1}\left(I_{m}\right),\right. \\
\widehat{\delta}(t) & \left.=\left[I-\left(\mathbf{H}(f)_{\Sigma}\right)^{*} \mathbf{H}(f)_{\Sigma}\right)\right]^{-1}\left(I_{n}\right),
\end{aligned}
$$

where $I_{k}$ stands for the constant matrix function on $\mathbb{R}$ with value $I_{k}$ for all $t \in \mathbb{R}$, and $\mathbf{H}(f)_{\Sigma}$ is seen as an operator acting $(B)_{[0, \infty) \cap \Sigma}^{n \times p} \rightarrow(B)_{(-\infty, 0] \cap \Sigma}^{m \times p}$ by applying $\mathbf{H}(f)_{\Sigma}$ to each column. Further, let

$$
\begin{aligned}
\alpha(t) & =\widehat{\alpha}(t) M\{\widehat{\alpha}\}^{-\frac{1}{2}}, \beta(t)=\widehat{\beta}(t) M\{\widehat{\delta}\}^{-\frac{1}{2}} \\
\gamma(t) & =\widehat{\gamma}(t) M\{\widehat{\alpha}\}^{-\frac{1}{2}}, \delta(t)=\widehat{\delta}(t) M\{\widehat{\delta}\}^{-\frac{1}{2}}
\end{aligned}
$$

Then the function

$$
g_{0}(t)=\beta(t) \delta(t)^{-1}=\left[\alpha(t)^{*}\right]^{-1} \gamma(t)^{*}, t \in \mathbb{R},
$$

is a strictly contractive extension in $(A P W)_{\Sigma}^{m \times n}$ of $f$.

It will be shown in the course of the proof of Theorem 4.1 that $\widehat{\alpha}$ is invertible in $(A P W)_{\Sigma}^{m \times m}$ and $M\{\widehat{\alpha}\}$ is positive definite. Similarly, it will be shown that $\widehat{\delta}$ is invertible in $(A P W)_{\Sigma}^{n \times n}$ and $M\{\widehat{\delta}\}>0$. Thus, the formulas (4.2)-(4.4) make sense. In fact, we shall show that $\widehat{\alpha}^{-1} \in(A P W)_{(-\infty, 0]}^{m \times m}$ and $\widehat{\delta}^{-1} \in(A P W)_{[0, \infty)}^{n \times n}$.

Next, we characterize the strictly contractive extension $g_{0}$ given by (4.4).

Theorem 4.2. Let $f \in(A P W)_{(-\infty, 0] \cap \Sigma}^{m \times n}$ be such that the conditions (i)-(iv) of Theorem 4.1 are satisfied (as in Theorem $4.1, \Sigma$ is an additive subgroup of $\mathbb{R}$ ). Let $g_{0}$ be given by (4.4). Then $g_{0}$ is the unique strictly contractive extension in $(A P W)_{\Sigma}^{m \times n}$ of $f$ such that

$$
g_{0}(t)\left(I_{n}-g_{0}(t)^{*} g_{0}(t)\right)^{-1}=\left(I_{m}-g_{0}(t) g_{0}(t)^{*}\right)^{-1} g_{0}(t) \in(A P W)_{(-\infty, 0] \cap \Sigma}^{m \times n} .
$$

Moreover, if $g$ is a strictly contractive extension in $(A P W)^{m \times n}$ of $f$, then

$$
\Delta\left(I_{n}-g_{0}(t)^{*} g_{0}(t)\right) \geq \Delta\left(I_{n}-g(t)^{*} g(t)\right),
$$

with equality if and only if $g(t)=g_{0}(t), t \in \mathbb{R}$. 
Here $\Delta(f)$ is the entropy (defined by $(3.6)$ ).

Finally, a parameterization of the set of all strictly contractive extensions in $(A P W)_{\Sigma}^{m \times n}$ of a function $f \in(A P W)_{(-\infty, 0] \cap \Sigma}^{m \times n}$ will be given. Introduce the parameter set

$$
(C A P W)_{(0, \infty) \cap \Sigma}^{m \times n}=\left\{g \in(A P W)_{(0, \infty) \cap \Sigma}^{m \times n}: \sup _{t \in \mathbb{R}}\|g(t)\|<1\right\} .
$$

Theorem 4.3. Let $\Sigma \subseteq \mathbb{R}$ be an additive subgroup, and let $f \in(A P W)_{(-\infty, 0] \cap \Sigma}^{m \times n}$ be such that $\left\|\mathbf{H}(f)_{\Sigma}\right\|<1$ (equivalently, $\left\|\mathbf{H}(f)_{\mathbb{R}}\right\|<1$ ). Define $\alpha(t), \beta(t), \gamma(t)$, and $\delta(t)$, as in Theorem 4.1. Then each strictly contractive extension in $(A P W)_{\Sigma}^{m \times n}$ of $f$ is of the form

$$
T(g)=(\alpha g+\beta)(\gamma g+\delta)^{-1}
$$

where $g \in(C A P W)_{(0, \infty) \cap \Sigma}^{m \times n}$. Also, this correspondence between $(C A P W)_{(0, \infty) \cap \Sigma}^{m \times n}$ and the set of strictly contractive extensions in $(A P W)_{\Sigma}^{m \times n}$ of $f$ is one-to-one.

Theorem 4.3 can be generalized in an obvious way. Namely, if $\Sigma^{\prime}$ is an additive subgroup of $\mathbb{R}$ that contains $\Sigma$, then the formula (4.7) provides a one-to-one correspondence between $g \in(C A P W)_{(0, \infty) \cap \Sigma^{\prime}}^{m \times n}$ and the set of strictly contractive extensions $T(g)$ in $(A P W)_{\Sigma^{\prime}}^{m \times n}$ of $f$. To obtain this generalization, apply Theorem 4.3 with $\Sigma$ replaced by $\Sigma^{\prime}$.

Of particular interest in Theorems 4.1-4.3 are the cases when $\Sigma=\mathbb{R}$ and when $\Sigma=\mathbb{Z}$. The former case corresponds to the Nehari problem stated in the introduction; the latter case is essentially the result for periodic matrix functions which dates back to [1].

The proofs of Theorems 4.1-4.3 will be given in Section 9 .

\section{Operators on the Besicovitch Space}

For the reader's convenience, we give in this section an exposition of several results on Besicovitch space operators and their relations to factorizations of almost periodic matrix valued functions.

We start with the definition of multiplication operators on the Besicovitch space. Let $a \in(A P)$. Define the operator $\mathbf{M}_{a}:(B) \rightarrow(B)$ by

$$
\left(\mathbf{M}_{a} f\right)(t)=a(t) f(t), \quad f \in(B) .
$$

More precisely, we define $\mathbf{M}_{a} f$ for $f \in(A P)$ using (5.1), and then extend $\mathbf{M}_{a}$ by continuity to the whole of $(B)$.

Next, we consider the connection with factorizations of almost periodic matrix functions. Let $G \in(A P)^{n \times n}$. We associate with $G$ two generalized Riemann operators:

$$
\mathbf{R}(G)=\Pi_{(0, \infty)}+\mathbf{M}_{G} \Pi_{(-\infty, 0]}:(B)^{n \times 1} \rightarrow(B)^{n \times 1}
$$

and

$$
\mathbf{R}^{\operatorname{var}}(G)=\Pi_{[0, \infty)}+\mathbf{M}_{G} \Pi_{(-\infty, 0)}:(B)^{n \times 1} \rightarrow(B)^{n \times 1}
$$

where

$$
\left(\mathbf{M}_{G} f\right)(x)=G(x) f(x), \quad f \in(B)^{n \times 1},
$$

is the matrix multiplication operator, which is introduced analogously to the scalar case (see (5.1)). 
Theorem 5.1. Assume that $G \in(A P W)^{n \times n}$. The following statements are equivalent:

(1) $\mathbf{R}(G)$ is an invertible operator on $(B)^{n \times 1}$;

(2) $\mathbf{R}^{\mathrm{var}}(G)$ is an invertible operator on $(B)^{n \times 1}$;

(3) G admits a factorization

where

$$
G(t)=G_{+}(t) G_{-}(t), \quad t \in \mathbb{R},
$$

$$
G_{+}^{ \pm 1} \in(A P)_{[0, \infty)}^{n \times n}, \quad G_{-}^{ \pm 1} \in(A P)_{(-\infty, 0]}^{n \times n}
$$

G admits a factorization (5.3), where

$$
G_{+}^{ \pm 1} \in(A P W)_{[0, \infty)}^{n \times n}, \quad G_{-}^{ \pm 1} \in(A P W)_{(-\infty, 0]}^{n \times n} .
$$

The equivalence of (1), (2) and (4) was established in [27]; the equivalence (3) $\Leftrightarrow(4)$ is contained in [37]. The corresponding statements (without proofs) can be found also in [28] (Theorems 3.4 and 3.5) and [30] (Lemma 5.5).

Representations (5.3) are called canonical factorizations of $G$; more about them in the next section.

We now turn our attention to the norms of generalized Hankel operators $\mathbf{H}(f)$ and

$$
\begin{aligned}
& \widetilde{\mathbf{H}}(f):(B)_{[0, \infty)}^{n \times 1} \rightarrow(B)_{(-\infty, 0)}^{m \times 1}, \quad g \mapsto \Pi_{(-\infty, 0)}(f g), \\
& \widetilde{\mathbf{H}}(f):(B)_{(0, \infty)}^{n \times 1} \rightarrow(B)_{(-\infty, 0]}^{m \times 1}, \quad g \mapsto \Pi_{(-\infty, 0]}(f g) .
\end{aligned}
$$

These operators are in some sense analogous to the more traditional Hankel operator

$$
\widehat{\mathbf{H}}(f):\left(H^{2+}\right)^{n \times 1} \rightarrow\left(H^{2-}\right)^{m \times 1}
$$

defined by the formula

$$
\widehat{\mathbf{H}}(f) g=\mathbf{P}_{-}(f g), \quad g \in\left(H^{2+}\right)^{n \times 1} .
$$

Here $\mathbf{P}_{-}$is the standard orthoprojection of $L^{2}(\mathbb{R})$ onto the Hardy space $H^{2-}$ in the lower half-plane. According to [1], for any $f \in\left(L^{\infty}\right)^{m \times n}$

$$
\|\widehat{\mathbf{H}}(f)\|=\inf _{\tilde{f} \in\left(H^{\infty}\right)^{m \times n}} \operatorname{ess} \sup _{t \in \mathbb{R}}\|f(t)+\widetilde{f}(t)\| .
$$

For $f \in(A P)^{m \times n}$ the latter formula can be supplemented by the following statement.

Theorem 5.2. Let $f \in(A P)^{m \times n}$. Then

$$
\begin{gathered}
\|\mathbf{H}(f)\|=\inf _{\tilde{f} \in(A P W)_{(0, \infty)}^{m \times n}} \sup _{t \in \mathbb{R}}\|f(t)+\widetilde{f}(t)\|, \\
\|\widehat{\mathbf{H}}(f)\|=\|\widetilde{\mathbf{H}}(f)\|=\|\widetilde{\widetilde{\mathbf{H}}}(f)\|=\inf _{\left.\widetilde{f} \in(A P W)_{(0, \infty)}^{m \times n}\right)} \sup _{t \in \mathbb{R}}\|f(t)+\widetilde{f}(t)\| .
\end{gathered}
$$

Proof. It was shown in [27] (see also Theorem 3.5 in [28]) that

$$
\left\|\Pi_{(-\infty, 0)} \mathbf{M}_{f} \Pi_{[0, \infty)}\right\|=\inf _{\tilde{f} \in(A P W)_{[0, \infty)}^{m \times n}} \sup _{t \in \mathbb{R}}\|f(t)+\widetilde{f}(t)\|
$$


and

$$
\left\|\Pi_{[0, \infty)} \mathbf{M}_{h} \Pi_{(-\infty, 0)}\right\|=\inf _{\tilde{h} \in(A P W)_{(-\infty, 0]}^{m \times n}} \sup _{t \in \mathbb{R}}\|h(t)+\widetilde{h}(t)\| .
$$

The formula for $\|\widetilde{\mathbf{H}}(f)\|$ follows directly from (5.8). To compute the norm of $\widetilde{\mathbf{H}}(f)$, we make use of the obvious relations $\Pi_{(-\infty, 0]}=\mathcal{C} \Pi_{[0, \infty)} \mathcal{C}, \Pi_{(0, \infty)}=\mathcal{C} \Pi_{(-\infty, 0)} \mathcal{C}$ (where $\mathcal{C}$ is the operator of complex conjugation), and of (5.9). Namely,

$$
\begin{aligned}
\|\widetilde{\mathbf{H}}(f)\| & =\left\|\Pi_{(-\infty, 0]} \mathbf{M}_{f} \Pi_{(0, \infty)}\right\|=\left\|\Pi_{[0, \infty)} \mathcal{C} \mathbf{M}_{f} \mathcal{C} \Pi_{(-\infty, 0)}\right\| \\
& =\left\|\Pi_{[0, \infty)} \mathbf{M}_{\bar{f}} \Pi_{(-\infty, 0)}\right\|=\inf _{\widetilde{h} \in(A P W)_{(-\infty, 0]}^{m \times n}} \sup _{t \in \mathbb{R}}\|\bar{f}(t)+\widetilde{h}(t)\| \\
& =\inf _{\tilde{f} \in(A P W)_{[0, \infty)}^{m \times n}} \sup _{t \in \mathbb{R}}\|\bar{f}(t)+\widetilde{\widetilde{f}}(t)\|=\inf _{\tilde{g} \in(A P W)_{[0, \infty)}^{m \times n}} \sup _{t \in \mathbb{R}}\|f(t)+\widetilde{g}(t)\| .
\end{aligned}
$$

Formula (5.6) can be established along the same lines as (5.8).

Finally, consider the map $\Psi$ defined by

$$
\Psi\left(\mathbf{P}_{-}\right)=\Pi_{(-\infty, 0)}, \quad \Psi\left(\mathbf{M}_{L, a}\right)=\mathbf{M}_{a}, a \in(A P),
$$

where $\mathbf{M}_{L, a}(f)=$ af is the multiplication operator acting on $\left(L^{2}(\mathbb{R})\right)$. It was shown in [26] that $\Psi$ can be extended to an isometric $*$-isomorphism from the $\mathrm{C}^{*}$ subalgebra of bounded linear operators on $\left(L^{2}(\mathbb{R})\right)$ generated by $\mathbf{P}_{-}$and $\mathbf{M}_{L, a}$, $a \in(A P)$, onto the corresponding $\mathrm{C}^{*}$-subalgebra of bounded linear operators on (B). From here it follows, in particular, that $\|\widehat{\mathbf{H}}(f)\|=\|\widetilde{\mathbf{H}}(f)\|$.

\section{Canonical Factorization of Almost Periodic Matrix Functions}

Let $G \in(A P)^{n \times n}$. A factorization (5.3) satisfying (5.4) is called a canonical factorization of $G$. Such factorizations, their generalizations and applications have been studied in $[37,30,29]$, for example. In this section we develop some results concerning canonical factorizations of a matrix function $G$ in the Wiener class, $G \in(A P W)^{n \times n}$. If the factors $G_{+}, G_{-}$are also in the Wiener class, that is, satisfy (5.5), we will call such canonical factorizations $W$-canonical factorizations.

Theorem 6.1. Assume that $G \in(A P W)^{n \times n}$ admits a $W$-canonical factorization. Let $\Sigma$ be the minimal additive subgroup of $\mathbb{R}$ that contains $\sigma(G)$. Then $G$ admits a $W$-canonical factorization (5.3) in which

$$
G_{+}^{ \pm 1} \in(A P W)_{[0, \infty) \cap \Sigma}^{n \times n}, \quad G_{-}^{ \pm 1} \in(A P W)_{(-\infty, 0] \cap \Sigma}^{n \times n} .
$$

Proof. Observe first of all that for any matrix function $F$ invertible in $(A P)^{n \times n}$ and such that $\sigma(F) \subseteq \Sigma$, also $\sigma\left(F^{-1}\right) \subseteq \Sigma$ (see Corollary 2.7). Therefore, we may suppose that $\sigma\left(G^{-1}\right) \subseteq \Sigma$, and we need to prove only that

$$
G_{+} \in(A P W)_{\Sigma}^{n \times n}
$$

The inclusions $G_{+}^{-1} \in(A P W)_{\Sigma}^{n \times n}$ and $G_{-}^{ \pm 1}=\left(G_{+}^{-1} G\right)^{ \pm 1} \in(A P W)_{\Sigma}^{n \times n}$ then follow automatically (cf. Proposition 2.2 (b)).

The key role in the proof of (6.1) is played by the formula

$$
G_{+}=\left(\mathbf{T}_{G}^{+}\right)^{-1}\left(I_{n}\right)
$$

(see [30, Lemma 5.7]), where

$$
\mathbf{T}_{G}^{+}=\Pi_{[0, \infty)} \mathbf{M}_{G^{-1}} \mid(B)_{[0, \infty)}^{n \times 1}
$$


is applied to the identity matrix $I_{n}$ columnwise. Consider some special cases first.

Case 1. Let $G$ be such that

$$
\left\|I-\mathbf{T}_{G}^{+}\right\|<1 .
$$

Then

$$
\left(\mathbf{T}_{G}^{+}\right)^{-1}=\sum_{k=0}^{\infty}\left(I-\mathbf{T}_{G}^{+}\right)^{k}
$$

and so

$$
G_{+}=\sum_{k=0}^{\infty}\left(I-\mathbf{T}_{G}^{+}\right)^{k}\left(I_{n}\right)
$$

Each term in the latter series belongs to $(A P W)_{\Sigma}^{n \times n}$. Since this series converges in $(B)^{n \times n}$, and its sum $G_{+}$a priori lies in $(A P W)_{[0, \infty)}^{n \times n}$, we conclude that

$$
G_{+} \in(B)_{\Sigma}^{n \times n} \cap(A P W)_{[0, \infty)}^{n \times n}=(A P W)_{\Sigma \cap[0, \infty)}^{n \times n} .
$$

Condition (6.2) is satisfied, in particular, if

$$
\left\|I-G^{-1}\right\|_{\infty}<1
$$

where $\|X\|_{\infty}$ stands for $\sup _{t \in \mathbb{R}}\|X(t)\|$. Indeed

$$
\left\|I-\mathbf{T}_{G}^{+}\right\|=\left\|\Pi_{[0, \infty)} \mathbf{M}_{I-G^{-1}} \mid(B)_{[0, \infty)}^{n \times n}\right\| \leq\left\|\mathbf{M}_{I-G^{-1}}\right\|,
$$

and for any $X \in(A P)^{n \times n}$ the norm of the multiplication operator $\mathbf{M}_{X}$ is equal to $\|X\|_{\infty}$.

Case 2. $G$ is hermitian positive definite. For such a $G$, consider $F(t)=$ $2\left\|G^{-1}\right\|_{\infty} G(t)$, which differs from $G$ by a scalar constant multiple. Obviously, it suffices to prove our statement for $F$. Observe that $F$ is hermitian positive definite along with $G$, and, in addition, all the eigenvalues (which in this case are the same as singular numbers) of $F^{-1}$ are located in a certain interval $[\epsilon, 1-\epsilon], \frac{1}{2}>\epsilon>0$. Then $\left\|I-F^{-1}\right\|_{\infty}<1$, and Case 1 can be applied.

Case 3. $G$ is unitary. Using the orthogonal decomposition

$$
(B)^{n \times 1}=(B)_{[0, \infty)}^{N \times 1} \oplus(B)_{(-\infty, 0)}^{n \times 1},
$$

write the multiplication operator $\mathbf{M}_{G^{-1}}\left(=\mathbf{M}_{G^{*}}\right)$ as

$$
\left[\begin{array}{cc}
\mathbf{T}_{G}^{+} & \mathbf{H}_{G}^{+} \\
\mathbf{H}_{G}^{-} & \mathbf{T}_{G}^{-}
\end{array}\right],
$$

where, naturally,

$$
\begin{aligned}
\mathbf{T}_{G}^{-} & =\Pi_{(-\infty, 0)} \mathbf{M}_{G^{-1}} \Pi_{(-\infty, 0)}, \\
\mathbf{H}_{G}^{+} & =\Pi_{[0,+\infty)} \mathbf{M}_{G^{-1}} \Pi_{(-\infty, 0)},
\end{aligned}
$$

and

$$
\mathbf{H}_{G}^{-}=\Pi_{(-\infty, 0)} \mathbf{M}_{G^{-1}} \Pi_{[0,+\infty)} .
$$


It is easy to check (see [7], where an abstract Hilbert space version of this statement is considered) that the operator $\mathbf{R}^{\operatorname{var}}(G)$ (defined by (5.2)) is invertible if and only if

$$
\left\|\mathbf{H}_{G}^{+}\right\|<1, \quad\left\|\mathbf{H}_{G}^{-}\right\|<1 .
$$

By Theorem 5.1 we know that inequalities (6.3) are valid. From (6.3) it follows that

$$
\begin{aligned}
{\left[\begin{array}{cc}
\mathbf{T}_{G}^{+} & 0 \\
0 & \mathbf{T}_{G}^{-}
\end{array}\right] } & =\mathbf{M}_{G^{-1}}-\left[\begin{array}{cc}
0 & \mathbf{H}_{G}^{+} \\
\mathbf{H}_{G}^{-} & 0
\end{array}\right] \\
& =\mathbf{M}_{G^{-1}}\left(I-\mathbf{M}_{G}\left[\begin{array}{cc}
0 & \mathbf{H}_{G}^{+} \\
\mathbf{H}_{G}^{-} & 0
\end{array}\right]\right) \\
& =M_{G^{-1}}(I-\Omega),
\end{aligned}
$$

where $\Omega=\mathbf{M}_{G}\left[\begin{array}{cc}0 & H_{G}^{+} \\ \mathbf{H}_{G}^{-} & 0\end{array}\right]$ is a contraction. Hence,

$$
\begin{aligned}
\left(\mathbf{T}_{G}^{+}\right)^{-1} & =\Pi_{[0, \infty)}(I-\Omega)^{-1} \mathbf{M}_{G} \mid(B)_{[0, \infty)}^{n \times 1} \\
& =\sum_{k=0}^{\infty} \Pi_{[0, \infty)} \Omega^{k} \mathbf{M}_{G} \mid(B)_{[0, \infty)}^{n \times 1},
\end{aligned}
$$

and the same reasoning as in Case 1 implies (6.1).

Finally, consider the general case. If $\sigma(G) \subseteq \Sigma$, then also $\sigma\left(G G^{*}\right) \subseteq \Sigma$. According to [37], an hermitian positive definite matrix $G G^{*}$ can be represented as $G G^{*}=A A^{*}$, where $A^{ \pm 1} \in(A P W)_{[0, \infty)}^{n \times n}$. As was shown in Case 2, in fact $A^{ \pm 1} \in(A P W)_{[0, \infty) \cap \Sigma}^{n \times n}$. Set $F=A^{-1} G$. Then $F=\left(A^{-1} G_{+}\right) G_{-}$is a representation (5.3) for $F$. On the other hand,

$$
F F^{*}=A^{-1} G G^{*} A^{*-1}=A^{-1} A A^{*} A^{*-1}=I,
$$

so that $F$ is unitary. Since $\sigma(F)$ is contained in $\Sigma$, together with $\sigma\left(A^{-1}\right)$ and $\sigma(G)$, we may use Case 3 to conclude that $\sigma\left(A^{-1} G_{+}\right) \subseteq \Sigma$. From here it follows that $\sigma\left(G_{+}\right)=\sigma\left(A \cdot\left(A^{-1} G_{+}\right)\right) \subseteq \Sigma$.

We also need a result on canonical factorization concerning the continuous dependence of the factors $G_{+}$and $G_{-}$on $G$. To make this notion precise, we normalize a canonical factorization (5.3) so that $G_{+}(0)=I$; then a canonical factorization, if it exists, is unique.

Theorem 6.2. Let $\Sigma$ be an additive subgroup in $\mathbb{R}$, and let $G_{\mu} \in(A P W)_{\Sigma}^{n \times n}$ be a family of matrix functions parameterized by $\mu \in[0,1]$. Assume that for every $\mu \in[0,1]$ there exists a normalized $W$-canonical factorization

$$
G_{\mu}(t)=G_{\mu+}(t) G_{\mu-}(t), \quad t \in \mathbb{R} .
$$

If $G_{\mu}$ is continuous as a function of $\mu$ in the Wiener norm

$$
\|X\|_{W}=\sum_{\lambda \in \sigma(X)}\left\|X_{\lambda}\right\|, \quad X(t)=\sum_{\lambda \in \sigma(X)} X_{\lambda} e^{i \lambda t} \in(A P W)^{n \times n},
$$


then the factors and their inverses $G_{\mu+}^{ \pm 1}$ and $G_{\mu-}^{ \pm 1}$ are also continuous in the same norm.

This result is contained in Theorem 2 in [37]. Observe that in view of Theorem 6.1 we must have (under the hypotheses of Theorem 6.2),

$$
G_{\mu+}^{ \pm 1} \in(A P W)_{[0, \infty) \cap \Sigma}^{n \times n}, \quad G_{\mu-}^{ \pm 1} \in(A P W)_{(-\infty, 0] \cap \Sigma}^{n \times n}
$$

We conclude this section with a result on $W$-canonical factorization of an hermitian valued function $G \in(A P W)^{n \times n}$. In this case, such a factorization exists in a special form that reflects the hermitian property of $G$ :

Theorem 6.3. Assume $G \in(A P W)^{n \times n}$, and assume that the matrix $G(t)$ is hermitian for every $t \in \mathbb{R}$. If $G$ admits a $W$-canonical factorization, then a $W$ canonical factorization of $G$ exists in the form

$$
G(t)=A_{+}(t)\left[\begin{array}{cc}
I_{p_{+}} & 0 \\
0 & -I_{p_{-}}
\end{array}\right]\left(A_{+}(t)\right)^{*},
$$

where $A_{+}^{ \pm 1} \in(A P W)_{[0, \infty) \cap \Sigma}^{n \times n}$, and where $\Sigma$ is the minimal additive subgroup of $\mathbb{R}$ that contains $\sigma(G)$. The sizes $p_{+}$and $p_{-}$are uniquely determined by $p_{+}+p_{-}=$ $n, p_{+}-p_{-}=\sigma$ (the signature of $G(t)$, which is constant for $t \in \mathbb{R}$ ).

Theorem 6.3 (without the property that $\sigma\left(A_{+}^{ \pm 1}\right) \subseteq \Sigma$ ) is proved in [37] (Theorem 3 there). The additional property $\sigma\left(A_{+}^{ \pm 1}\right) \subseteq \Sigma$ is obtained by repeating the proof of Theorem 3 in [37] using Theorem 6.1.

\section{Invertibility of Elements in Banach Algebras}

We prove here a general result on invertibility in Banach algebras which later on will be specialized to the algebras of the form $(A P W)_{\Sigma}^{n \times n}$, for suitable sets $\Sigma \subseteq \mathbf{R}$.

Theorem 7.1. Let $\mathcal{B}$ be a unital Banach algebra. Let $\Omega \subseteq \mathcal{B}$ be a connected set such that for every $x \in \Omega$ there is an $\alpha>0$ such that

$$
\|x y\|>\alpha\|y\| \quad \text { for every } y \in \mathcal{B}, y \neq 0 .
$$

If some element $x_{0} \in \Omega$ is invertible in $\mathcal{B}$, then every element $x \in \Omega$ is invertible in $\mathcal{B}$.

Proof. Consider the regular representation $x \mapsto \Phi(x), x \in \mathcal{B}$, where $\Phi(x)$ is an operator on $\mathcal{B}$ defined by $\Phi(x)(y)=x y, y \in \mathcal{B}$. By the condition (7.1), clearly $\operatorname{Ker} \Phi(x)=\{0\}$ and $\operatorname{Im} \Phi(x)$ is closed for every $x \in \Omega$. By the perturbation theory of linear operators (Kato's theorem, see, e.g., Theorem V.1.6 in [23]), the index

$$
\operatorname{dim} \operatorname{Ker} \Phi(x)-\operatorname{codim} \operatorname{Im} \Phi(x)=-\operatorname{codim} \operatorname{Im} \Phi(x) \geq-\infty
$$

is constant for $x \in \Omega$. Since $\Phi\left(x_{0}\right)$ is invertible, it follows that codim $\operatorname{Im} \Phi(x)=0$ for all $x \in \Omega$, and hence $\Phi(x)$ is invertible (as an operator on $\mathcal{B}$ ) for all $x \in \Omega$.

Let $\Phi(\mathcal{B})=\{\Phi(x): x \in \mathcal{B}\}$. Clearly, $\Phi(\mathcal{B})$ is a norm closed unital subalgebra in the algebra of all linear bounded operators on $\mathcal{B}$. Let

$$
\Omega_{0}=\left\{x \in \Omega \mid \text { the inverse } \Phi(x)^{-1} \text { belongs to } \Phi(\mathcal{B})\right\} .
$$

Since the set of invertible elements in $\Phi(\mathcal{B})$ is open, the set $\Omega_{0}$ is open in $\Omega$ as well. But $\Omega_{0}$ is also closed in $\Omega$. Indeed, let $\left\{x_{q}\right\}_{q=1}^{\infty}$ be a sequence in $\Omega_{0}$ such that $x_{q} \rightarrow x \in \Omega$. Since $\Phi\left(x_{q}\right)$ and $\Phi(x)$ are invertible, we have $\Phi\left(x_{q}\right)^{-1} \rightarrow \Phi(x)^{-1}$. But $\Phi\left(x_{q}\right)^{-1} \in \Phi(\mathcal{B})$ and $\Phi(\mathcal{B})$ is norm closed. Hence also $\Phi(x)^{-1} \in \Phi(\mathcal{B})$, so $x \in \Omega_{0}$. 
Now $\Omega_{0} \neq \emptyset$ (because $x_{0} \in \Omega_{0}$ ), and we conclude that $\Omega_{0}=\Omega$, which proves the theorem.

We apply Theorem 7.1 in the following situation. Let $\Sigma \subseteq \mathbb{R}$ be a set containing zero such that $\alpha, \beta \in \Sigma \Rightarrow \alpha+\beta \in \Sigma$. Then $(A P W)_{\Sigma}^{n \times n}$ is a closed unital subalgebra of $(A P W)^{n \times n}$ (see Proposition $2.4(\mathrm{~b})$ ).

Theorem 7.2. Let $\Sigma$ be as above. Let $\Omega \subseteq(A P W)_{\Sigma}^{n \times n}$ be a connected set in the Wiener norm (6.4) such that

$$
\inf _{t \in \mathbb{R}}|\operatorname{det} F(t)|>0
$$

for every $F \in \Omega$. If there exists $G_{0} \in \Omega$ which is invertible in $(A P W)_{\Sigma}^{n \times n}$, then every $G \in \Omega$ is invertible in $(A P W)_{\Sigma}^{n \times n}$.

Proof. Consider the Banach algebra $\mathcal{B}=(A P W)_{\Sigma}^{n \times n}$ with the Wiener norm $\|\cdot\|_{W}$. By the hypothesis (7.2) every $F \in \Omega$ has an inverse $F^{-1} \in(A P W)^{n \times n}$ (cf. (2.2)). Therefore for $F \in \Omega$ and $G \in(A P W)_{\Sigma}^{n \times n}$ we have:

$$
\|G\|_{W}=\left\|F^{-1} \cdot F G\right\|_{W} \leq\left\|F^{-1}\right\|_{W} \cdot\|F G\|_{W} .
$$

Thus, the condition (7.1) is satisfied, and Theorem 7.1 is applicable.

\section{Proofs of the Main Results on the Positive Extension Problem}

In this section we prove Theorems 3.1-3.3. Throughout this section $\Sigma \subseteq \mathbb{R}$ is an additive subgroup, and $\mu \in \Sigma, \mu>0$.

We focus first on Theorem 3.1, and start with the relatively easy parts of the theorem.

The equivalence of (ii) and (iii) (as well as of (v) and (vi)), is obvious in view of the unitary operator $\Phi:(B)^{n \times 1} \rightarrow(B)^{n \times 1}$ defined by $(\Phi f)(x)=f(-x), f \in$ $(A P W)^{n \times 1}$, which transforms $\mathbf{T}(f)_{[0, \mu] \cap \Sigma}$ to $\mathbf{T}(f)_{[-\mu, 0] \cap \Sigma}$. The implication (i) $\Rightarrow$ (ii) (and, for the similar reasons, (iv) $\Rightarrow(\mathrm{v})$ ) is also clear. Indeed, let $h$ be a positive extension in $(A P W)_{\Sigma}^{n \times n}$ of $f$, as in (i). Then $\mathbf{T}(f)_{[0, \mu] \cap \Sigma}=\Pi_{[0, \mu]} M_{h} \Pi_{[0, \mu]}$, where $M_{h} g=h g$ is the multiplication operator on $(B)_{\Sigma}^{n \times 1}$, and $M_{h}$ is positive definite because $h$ is positive.

The equivalence of (ii) and (v) is seen from the following lemma.

Lemma 8.1. Let $f \in(A P W)_{[-\mu, \mu] \cap \Sigma}^{n \times n}$. If one of the operators $\mathbf{T}(f)_{[0, \mu]}$ and $\mathbf{T}(f)_{[0, \mu] \cap \Sigma}$ is positive definite, then the other is also positive definite. Moreover, $\mathbf{T}(f)_{[0, \mu]} \geq \epsilon I$ holds for some $\epsilon>0$ if and only if $\mathbf{T}(f)_{[0, \mu] \cap \Sigma} \geq \epsilon I$ holds.

In turn, Lemma 8.1 is a corollary of a more precise result:

Lemma 8.2. Let $f \in(A P W)_{[-\mu, \mu] \cap \Sigma}^{n \times n}$. Let $\Omega$ be a set having the cardinality of the set of nontrivial (i.e., different from $\Sigma$ itself) cosets of $\Sigma$ in $\mathbb{R}$. Then there exists an increasing sequence of closed subspaces $D_{1} \subseteq D_{2} \subseteq \cdots$ in $(B)_{[0, \mu]}^{n \times 1}$ whose union coincides with $(B)_{[0, \mu]}^{n \times 1}$, having the following property: Let $P_{j}$ be the orthogonal projection on $D_{j}(j=1,2, \ldots)$. Then for each $j$, the compression $P_{j} \mathbf{T}(f)_{[0, \mu]} P_{j}$ : $D_{j} \rightarrow D_{j}$ is unitarily similar to an orthogonal sum of the form

$$
\left(\mathbf{T}(f)_{[0, \mu] \cap \Sigma}\right) \oplus \bigoplus_{\omega \in \Omega} Q_{\omega, j}\left(\mathbf{T}(f)_{[0, \mu] \cap \Sigma}\right) Q_{\omega, j},
$$


where $Q_{\omega, j}$ is an orthogonal projection on some subspace in $(B)_{[0, \mu] \cap \Sigma}^{n \times 1}$, and

$$
Q_{\omega, j}\left(\mathbf{T}(f)_{[0, \mu] \cap \Sigma}\right) Q_{\omega, j}
$$

is understood as an operator on the range of $Q_{\omega, j}$.

Proof. Let $\mathbb{R}=\Sigma \cup \bigcup_{\omega \in \Omega} \Sigma_{\omega}$ be the representation of $\mathbb{R}$ as a disjoint union of cosets of $\Sigma$, where the cosets $\Sigma_{\omega}$ are indexed by $\omega \in \Omega$, and $\Sigma_{\omega} \neq \Sigma, \omega \in \Omega$. We distinguish two cases: (1) $\Sigma$ is a discrete set (in this case there exists a minimal positive element in $\Sigma$ ); (2) $\Sigma$ is a dense set (in this case there does not exist a minimal positive element in $\Sigma$ ).

We consider case (2) first. For every $\Sigma_{\omega}$, select a decreasing sequence of positive elements $\left\{r_{\omega, m}\right\}_{m=1}^{\infty}$ in $\Sigma_{\omega}$ such that $r_{\omega, m}<\mu$ for all $m$, and $r_{\omega, m}<\frac{1}{m}$ (in particular, $r_{\omega, m} \rightarrow 0$ as $\left.m \rightarrow \infty\right)$. Let

$$
D_{m}=(B)_{[0, \mu] \cap \Gamma_{m}}^{n \times 1}
$$

where $\Gamma_{m}=\Sigma \cup \bigcup_{\omega \in \Omega}\left(\left[r_{\omega, m}, \mu\right] \cap \Sigma_{\omega}\right)$. Clearly, $D_{m}$ is closed. Because of the selection of the numbers $\left\{r_{\omega, m}\right\}_{m=1}^{\infty}$ it is also clear that $\left\{D_{m}\right\}_{m=1}^{\infty}$ is an increasing sequence of subspaces whose union is $(B)_{[0, \mu]}^{n \times 1}$. Furthermore, $D_{m}$ has the orthogonal decomposition

$$
D_{m}=(B)_{[0, \mu] \cap \Sigma}^{n \times 1} \oplus \bigoplus_{\omega \in \Omega}(B)_{\left[r_{\omega, m}, \mu\right] \cap \Sigma_{\omega}}^{n \times 1} .
$$

We also have the orthogonal decomposition of the vector Besicovitch space:

$$
(B)_{[0, \mu]}^{n \times 1}=(B)_{[0, \mu] \cap \Sigma}^{n \times 1} \oplus \bigoplus_{\omega \in \Omega}(B)_{[0, \mu] \cap \Sigma_{\omega}}^{n \times 1} .
$$

Since $\Sigma$ is an additive subgroup, the multiplication formula (2.1) easily implies that each $(B)_{[0, \mu] \cap \Sigma_{\omega}}^{n \times 1}$ is invariant for $\mathbf{T}(f)_{[0, \mu]}$.

Now fix $\omega \in \Omega$ and a positive integer $m$, and consider the unitary operator

$$
\Phi_{\omega, m}:(B)_{\left[r_{\omega, m}, \mu\right] \cap \Sigma_{\omega}}^{n \times 1} \rightarrow(B)_{\left[0, \mu-r_{\omega, m}\right] \cap \Sigma}^{n \times 1}
$$

defined by $\left(\Phi_{\omega, m} g\right)(t)=e^{-i r_{\omega, m} t} g(t)$. We then have for every $g \in(B)_{\left[r_{\omega, m}, \mu\right] \cap \Sigma_{\omega}}^{n \times 1}$ :

$$
\begin{aligned}
\Pi_{\left[r_{\omega, m}, \mu\right]} \mathbf{T}(f)_{[0, \mu]} g & =\Pi_{\left[r_{\omega, m}, \mu\right]}(f g) \\
& =\Pi_{\left[r_{\omega, m}, \mu\right]}\left(e^{i r_{\omega, m} t} f\left(\Phi_{\omega, m} g\right)\right) \\
& =\left(\Phi_{\omega, m}\right)^{-1}\left(\Pi_{\left[0, \mu-r_{\omega, m}\right]} f\left(\Phi_{\omega, m} g\right)\right) \\
& \left.=\left(\Phi_{\omega, m}\right)^{-1} \mathbf{T}(f)_{\left[0, \mu-r_{\omega, m}\right] \cap \Sigma}\left(\Phi_{\omega, m} g\right)\right) .
\end{aligned}
$$

Thus, the operator

$$
\Pi_{\left[r_{\omega, m}, \mu\right]} \mathbf{T}(f)_{[0, \mu]} \Pi_{\left[r_{\omega, m}, \mu\right]}:(B)_{\left[r_{\omega, m}, \mu\right] \cap \Sigma_{\omega}}^{n \times 1} \rightarrow(B)_{\left[r_{\omega, m}, \mu\right] \cap \Sigma_{\omega}}^{n \times 1}
$$

is unitarily similar to the operator

$$
\Pi_{\left[0, \mu-r_{\omega, m}\right]} \mathbf{T}(f)_{[0, \mu] \cap \Sigma} \Pi_{\left[0, \mu-r_{\omega, m}\right]}: \quad(B)_{\left[0, \mu-r_{\omega, m}\right] \cap \Sigma}^{n \times 1} \rightarrow(B)_{\left[0, \mu-r_{\omega, m}\right] \cap \Sigma}^{n \times 1}
$$


Using the orthogonal decompositions (8.2) and (8.3), and denoting by $P_{m}$ the orthogonal projection on $D_{m}$, we obtain that $P_{m} \mathbf{T}(f)_{[0, \mu]} P_{m}$ indeed has the required form (8.1).

Consider now the case when $\Sigma$ is a discrete set. Let $r_{\omega}$ be the smallest positive number (necessarily smaller than $\mu$ ) in the coset $\Sigma_{\omega}$. We have then the orthogonal decomposition

$$
(B)_{[0, \mu]}^{n \times 1}=(B)_{[0, \mu] \cap \Sigma}^{n \times 1} \oplus \bigoplus_{\omega \in \Omega}(B)_{\left[r_{\omega}, \mu\right] \cap \Sigma_{\omega}}^{n \times 1},
$$

and, arguing as above, it follows that the operator $\mathbf{T}(f)_{[0, \mu]}$ itself is unitarily similar to an orthogonal sum of the form (8.1).

To prove the implication (v) $\Rightarrow$ (i) (the difficult part of Theorem 3.1), we employ the band method, as in [38]. A preliminary result is needed:

Lemma 8.3. Let $f \in(A P W)_{[-\mu, \mu] \cap \Sigma}^{n \times n}$ be such that $\mathbf{T}(f)_{[0, \mu]}>0$ (equivalently, by Lemma 8.1, $\left.\mathbf{T}(f)_{[0, \mu] \cap \Sigma}>0\right)$. Then there exist unique $x, y \in(A P W)_{\Sigma}^{n \times n}$ such that

(1) $\sigma(x) \subseteq[0, \mu] \cap \Sigma, \sigma(y) \subseteq[-\mu, 0] \cap \Sigma$;

(2) $\sigma(f x-I) \subseteq((-\infty, 0) \cup(\mu, \infty)) \cap \Sigma$, $\sigma(f y-I) \subseteq((-\infty,-\mu) \cup(0, \infty)) \cap \Sigma$.

In fact, $x$ and $y$ are given by the formula (3.4). Moreover, $x$ and $y$ are invertible in $(A P W)_{\Sigma}^{n \times n}$, and

(3) $\sigma\left(x^{-1}\right) \subseteq[0, \infty) \cap \Sigma, \sigma\left(y^{-1}\right) \subseteq(-\infty, 0] \cap \Sigma$;

(4) $M\{x\}>0, \quad M\{y\}>0$.

Proof. We shall prove the existence of $x$. The existence of $y$ can be shown in a similar way. Consider

$$
G(t)=\left[\begin{array}{cc}
f(t) & e^{-i \mu t} I_{n} \\
e^{i \mu t} I_{n} & 0
\end{array}\right] \in(A P W)_{\Sigma}^{2 n \times 2 n} .
$$

A straightforward matrix generalization of Lemma 1.3 in [38] shows that positivity of $\mathbf{T}(f)_{[0, \mu]}$ implies invertibility of $\mathbf{R}(G)$ (a variation of this result is discussed in Section 10; see Lemma 10.1 and its proof). From here and Theorem 5.1 it follows that $G(t)$ admits a $W$-canonical factorization. Being hermitian with $\operatorname{det} G(t) \equiv$ $(-1)^{n}$ and an equal number of positive and negative eigenvalues, this matrix can be represented in a form of Theorem 6.3:

$$
G(t)=A(t) J A(t)^{*}
$$

where $\operatorname{det} A(t) \equiv 1, J=\left[\begin{array}{cc}I_{n} & 0 \\ 0 & -I_{n}\end{array}\right], A(t)^{ \pm 1} \in(A P W)_{[0, \infty) \cap \Sigma}^{2 n \times 2 n}$. Denote

$$
A(t)=\left[\begin{array}{ll}
\alpha(t) & \beta(t) \\
\gamma(t) & \delta(t)
\end{array}\right]
$$

with $\alpha, \beta, \gamma, \delta \in(A P W)_{[0, \infty) \cap \Sigma}^{n \times n}$. Consider the Riemann boundary value problem

$$
\left[\begin{array}{c}
x_{2}^{+} \\
x_{1}^{+}
\end{array}\right]=G\left[\begin{array}{c}
x_{1}^{-} \\
x_{2}^{-}
\end{array}\right]
$$


with $x_{i}^{ \pm} \in(A P W)_{ \pm[0, \infty) \cap \Sigma}^{n \times n}, i=1,2$. Using the factorization (8.4), we may rewrite (8.5) as

$$
A^{-1}\left[\begin{array}{c}
x_{2}^{+} \\
x_{1}^{+}
\end{array}\right]=J A^{*}\left[\begin{array}{l}
x_{1}^{-} \\
x_{2}^{-}
\end{array}\right] .
$$

As the left-hand side of $(8.6)$ has spectrum in $[0, \infty)$ and the right-hand side has spectrum in $(-\infty, 0]$, both sides of $(8.6)$ are constant, say equal to $\left[\begin{array}{l}c_{1} \\ c_{2}\end{array}\right]$. The general solution of (8.5) therefore is given by

$$
\left[\begin{array}{c}
x_{2}^{+} \\
x_{1}^{+}
\end{array}\right]=A\left[\begin{array}{l}
c_{1} \\
c_{2}
\end{array}\right], \quad\left[\begin{array}{l}
x_{1}^{-} \\
x_{2}^{-}
\end{array}\right]=A^{*-1} J\left[\begin{array}{l}
c_{1} \\
c_{2}
\end{array}\right], \quad c_{1}, c_{2} \in \mathbb{C}^{n \times n} .
$$

As $\operatorname{det} A(t) \equiv 1$, and $A(t) \in(A P W)_{[0, \infty) \cap \Sigma}^{2 n \times 2 n}$, we also have that $\operatorname{det} M\{A\}=1$ (because $M\{\cdot\}$ is an additive and multiplicative functional on $(A P W)_{[0, \infty) \cap \Sigma}$; see Proposition 2.4 (c)). Denote $\alpha_{0}=M\{\alpha\}, \beta_{0}=M\{\beta\}, \gamma_{0}=M\{\gamma\}$, and $\delta_{0}=M\{\delta\}$. Then

$$
\operatorname{det}\left[\begin{array}{cc}
\alpha_{0} & \beta_{0} \\
\gamma_{0} & \delta_{0}
\end{array}\right]=1
$$

so

$$
\left[\begin{array}{ll}
\alpha_{0} & \beta_{0} \\
\gamma_{0} & \delta_{0}
\end{array}\right]
$$

is invertible. Let

$$
\left[\begin{array}{ll}
p_{0} & q_{0} \\
r_{0} & s_{0}
\end{array}\right]=\left[\begin{array}{ll}
\alpha_{0} & \beta_{0} \\
\gamma_{0} & \delta_{0}
\end{array}\right]^{-1}
$$

Now let $c_{1}=q_{0}$ and $c_{2}=s_{0}$, and define $x_{1}^{+}, x_{2}^{+}, x_{1}^{-}, x_{2}^{-}$by (8.7). Then

$$
\left\{\begin{array}{l}
M\left\{x_{1}^{+}\right\}=M\left\{\gamma q_{0}+\delta s_{0}\right\}=\gamma_{0} q_{0}+\delta_{0} s_{0}=I_{n} \\
M\left\{x_{2}^{+}\right\}=M\left\{\alpha q_{0}+\beta s_{0}\right\}=\alpha_{0} q_{0}+\beta_{0} s_{0}=0
\end{array}\right.
$$

and by (8.5) we get that

$$
\begin{aligned}
x_{1}^{+*} f x_{1}^{+} & =x_{1}^{+*}\left(e^{i \mu t} x_{2}^{+}-x_{2}^{-}\right) \\
& =x_{1}^{-*} x_{2}^{+}-x_{1}^{+*} x_{2}^{-} .
\end{aligned}
$$

The latter equality yields that

$$
M\left\{x_{1}^{+*} f x_{1}^{+}\right\}=M\left\{x_{1}^{-*}\right\} M\left\{x_{2}^{+}\right\}-M\left\{x_{1}^{+*}\right\} M\left\{x_{2}^{-}\right\},
$$

where we have again used the multiplicative properties of $M\{\cdot\}$ on $(A P W)_{(-\infty, 0]}$ and on $(A P W)_{[0, \infty)}$. By (8.8) $M\left\{x_{2}^{+}\right\}=0$, so we get

$$
M\left\{x_{1}^{+*} f x_{1}^{+}\right\}=-M\left\{x_{1}^{+*}\right\} M\left\{x_{2}^{-}\right\} .
$$

Observe that (in view of $\sigma(f) \subseteq[-\mu, \mu], \sigma\left(x_{1}^{+}\right) \subseteq[0, \infty)$ )

$$
\left(x_{1}^{+*} f x_{1}^{+}\right)_{0}=\left(\left(\Pi_{[0, \mu]} x_{1}^{+}\right)^{*} f\left(\Pi_{[0, \mu]} x_{1}^{+}\right)\right)_{0} ;
$$


use the multiplication formula (2.1) with $\lambda=0$ to verify this equality. Next we obtain that

$$
M\left\{x_{1}^{+*} f x_{1}^{+}\right\}>0
$$

Indeed, if $v \in \mathbb{C}^{n}$, then

$$
\begin{aligned}
v^{*} M\left\{x_{1}^{+*} f x_{1}^{+}\right\} v & =M\left\{v^{*} x_{1}^{+*} f x_{1}^{+} v\right\} \\
& =\left\langle\mathbf{T}(f)_{[0, \mu]} x_{1}^{+} v, x_{1}^{+} v\right\rangle \\
& \geq \epsilon\left\langle x_{1}^{+} v, x_{1}^{+} v\right\rangle \\
& =\epsilon M\left\{v^{*} x_{1}^{+*} x_{1}^{+} v\right\} \\
& =\epsilon v^{*} M\left\{x_{1}^{+*} x_{1}^{+}\right\} v \geq \epsilon v^{*} v
\end{aligned}
$$

where $\epsilon>0$ depends only on $f$. Note that we have used the equalities $\Pi_{[0, \mu]} x_{1}^{+}=x_{1}^{+}$ and $M\left\{x_{1}^{+}\right\}=I_{n}$ (yielding $M\left\{x_{1}^{+*} x_{1}^{+}\right\} \geq I$ ). Thus, by (8.9), we obtain

$$
-M\left\{x_{1}^{+*}\right\} M\left\{x_{2}^{-}\right\}>0 .
$$

In particular, $M\left\{x_{2}^{-}\right\}$is invertible.

Now let

$$
\widehat{c}_{1}=-c_{1} M\left\{x_{2}^{-}\right\}^{-1}, \quad \widehat{c}_{2}=-c_{2} M\left\{x_{2}^{-}\right\}^{-1},
$$

and put

$$
x=\gamma \widehat{c}_{1}+\delta \widehat{c}_{2} \quad\left(=-x_{1}^{+} M\left\{x_{2}^{-}\right\}^{-1}\right) .
$$

Then $\sigma(x)=\sigma\left(-x_{1}^{+} M\left\{x_{2}^{-}\right\}^{-1}\right) \subseteq[0, \mu]$ (this containment follows from $x_{1}^{+}=$ $e^{i \mu t} x_{1}^{-}$; (see (8.5)), and

$$
\begin{aligned}
\sigma(f x-I) & =\sigma\left(-f x_{1}^{+} M\left\{x_{2}^{-}\right\}^{-1}-I\right) \\
& =\sigma\left(\left(-e^{i \mu t} x_{2}^{+}+x_{2}^{-}-M\left\{x_{2}^{-}\right\}\right) M\left\{x_{2}^{-}\right\}^{-1}\right) \\
& \subseteq(-\infty, 0) \cup(\mu, \infty),
\end{aligned}
$$

where we use that $M\left\{x_{2}^{+}\right\}=0$ by (8.8). Furthermore,

$$
M\{x\}=-M\left\{x_{1}^{+}\right\} M\left\{x_{2}^{-}\right\}^{-1}=-M\left\{x_{2}^{-}\right\}^{-1}>0
$$

by (8.10) and taking into account that $M\left\{x_{2}^{+}\right\}=I$.

It remains to show that $x$ is invertible in $(A P W)^{n \times n}$ and $\sigma\left(x^{-1}\right) \subseteq[0, \infty) \cap \Sigma$. This will be done in two steps. First, we show that $x$ is invertible in $(A P W)^{n \times n}$. Consider

$$
\widehat{c}_{2}^{*} \widehat{c}_{2}-\widehat{c}_{1}^{*} \widehat{c}_{1}=M\left\{x_{2}^{-}\right\}^{-1 *}\left(c_{2}^{*} c_{2}-c_{1}^{*} c_{1}\right) M\left\{x_{2}^{-}\right\}^{-1},
$$


and note that

$$
\begin{aligned}
c_{1}^{*} c_{1}-c_{2}^{*} c_{2} & =\left[c_{1}^{*} c_{2}^{*}\right] J\left[\begin{array}{l}
c_{1} \\
c_{2}
\end{array}\right] \\
& =\left[c_{1}^{*} c_{2}^{*}\right] A^{*}\left[\begin{array}{l}
x_{1}^{-} \\
x_{2}^{-}
\end{array}\right] \\
& =c_{1}^{*}\left(\alpha^{*} x_{1}^{-}+\gamma^{*} x_{2}^{-}\right)+c_{2}^{*}\left(\beta^{*} x_{1}^{-}+\delta^{*} x_{2}^{-}\right) .
\end{aligned}
$$

Since the left-hand side is constant, we must have (using the multiplicativity of $M\{\cdot\}$ on $(A P W)_{(-\infty, 0]}^{n \times n}$ and the inequality (8.10))

$$
\begin{aligned}
c_{1}^{*} c_{1}-c_{2}^{*} c_{2} & =M\left\{c_{1}^{*}\left(\alpha^{*} x_{1}^{-}+\gamma^{*} x_{2}^{-}\right)+c_{2}^{*}\left(\beta^{*} x_{1}^{-}+\delta^{*} x_{2}^{-}\right)\right\} \\
& =\left(c_{1}^{*} \alpha_{0}^{*}+c_{2}^{*} \beta_{0}^{*}\right) M\left\{x_{1}^{-}\right\}+\left(c_{1}^{*} \gamma_{0}^{*}+c_{2}^{*} \delta_{0}^{*}\right) M\left\{x_{2}^{-}\right\} \\
& =M\left\{x_{1}^{+*}\right\} M\left\{x_{2}^{-}\right\}<0 .
\end{aligned}
$$

Consequently,

$$
\widehat{c}_{2}^{*} \widehat{c}_{2}>\widehat{c}_{1}^{*} \widehat{c}_{1}+\epsilon_{0} I
$$

for some positive $\epsilon_{0}$. In particular, $\widehat{c}_{2}$ is invertible in $(A P W)^{n \times n}$. Also note that $\gamma \gamma^{*}=\delta \delta^{*}$ in view of (8.4). Moreover, there exists an $\epsilon>0$ such that every eigenvalue of $\gamma(t) \gamma(t)^{*}$ is not smaller than $\epsilon$, for every $t \in \mathbb{R}$. Indeed, otherwise there would be a sequence $\left\{t_{p}\right\}_{p=1}^{\infty} \in \mathbb{R}$ and a sequence of unit length row vectors $\left\{u_{p}\right\}_{p=1}^{\infty} \in \mathbb{C}^{1 \times n}$ such that $\left\|u_{p} \gamma\left(t_{p}\right)\right\|=\left\|u_{p} \delta\left(t_{p}\right)\right\| \rightarrow 0$ as $p \rightarrow \infty$. Then

$$
\left\|\left[\begin{array}{ll}
0 & u_{p}
\end{array}\right] A\left(t_{p}\right) A\left(t_{p}\right)^{*}\left[\begin{array}{c}
0 \\
u_{p}^{*}
\end{array}\right]\right\| \rightarrow 0 \quad \text { as } p \rightarrow \infty,
$$

because $A(t)^{*} \in(A P W)^{2 n \times 2 n}$ and in particular $\sup _{t \in \mathbb{R}}\left\|A(t)^{*}\right\|<\infty$. Now (8.13) implies, together with $\operatorname{det}\left(A(t) A(t)^{*}\right) \equiv 1$, that the largest eigenvalue of $A(t) A(t)^{*}$ is unbounded for $t \in \mathbb{R}$. It follows that the norm of $A(t) A(t)^{*}$ is unbounded as well, a contradiction with $A(t) A(t)^{*} \in(A P W)^{2 n \times 2 n}$. Now by Proposition 2.3, the matrix function $H(t)=\left(\gamma(t) \gamma(t)^{*}\right)^{\frac{1}{2}}$ and its inverse $H(t)^{-1}$ belong to $(A P W)^{n \times n}$. Hence in the polar decompositions $\gamma=H U_{1}, \delta=H U_{2}$, where $U_{1}$ and $U_{2}$ are unitary, we also have $U_{1}, U_{2} \in(A P W)^{n \times n}$. Write

$$
x=H\left(U_{1} \widehat{c}_{1}+U_{2} \widehat{c}_{2}\right)=H U_{2}\left(U_{2}^{-1} U_{1} \widehat{c}_{1} \widehat{c}_{2}^{-1}+I\right) \widehat{c}_{2},
$$

and since $U_{2}^{-1} U_{1} \widehat{c}_{1} \widehat{c}_{2}^{-1}$ is a strict contraction in view of (8.12), the matrix function $x$ is invertible in $(A P W)^{n \times n}$ (cf. (2.2)).

To prove that $\sigma\left(x^{-1}\right) \subseteq[0, \infty) \cap \Sigma$, we consider a family of functions

$$
f^{(\alpha)}=\alpha I_{n}+(1-\alpha) f, \quad 0 \leq \alpha \leq 1 \text {. }
$$

Clearly, $\mathbf{T}\left(f^{(\alpha)}\right)_{[0, \mu]}=\alpha I+(1-\alpha) \mathbf{T}(f)_{[0, \mu]}>0$ for all $\alpha \in[0,1]$. Define $x^{(\alpha)}$ by the formula (8.11) (or equivalently, (3.4)), with $f$ replaced by $f^{(\alpha)}$. Then $x^{(\alpha)} \in(A P W)_{[0, \infty) \cap \Sigma}^{n \times n}$. By Theorem $6.2, x^{(\alpha)}$ is a continuous function of $\alpha$. Obviously, $x^{(0)}=x$, and a computation shows that $x^{(1)}=I_{n}$ (in this case take $\left.A(t)=\left[\begin{array}{cc}I_{n} & 0 \\ e^{i \mu t} I_{n} & I_{n}\end{array}\right]\right)$. Also, by the already proved part of Lemma $8.3 x^{(\alpha)}$ is 
invertible in $(A P W)^{n \times n}$ for all $\alpha \in[0,1]$. Now by Theorem 7.2 we have that $x^{-1} \in(A P W)_{[0, \infty) \cap \Sigma}^{n \times n}$.

Finally, for the uniqueness of $x$ with the requisite properties, observe that $\sigma(x) \subseteq$ $[0, \mu] \cap \Sigma$ implies that $x \in(A P W)_{[0, \mu] \cap \Sigma}^{n \times n}$. But then the $j^{\text {th }}$ column $x_{j}$ of $x$ belongs to $(B)_{[0, \mu] \cap \Sigma}^{n \times 1}$. The condition

$$
\sigma(f x-I) \subseteq(-\infty, 0) \cup(\mu, \infty)
$$

implies that

$$
\Pi_{[0, \mu]}(f x)=I .
$$

Consequently,

$$
\mathbf{T}(f)_{[0, \mu] \cap \Sigma}\left(x_{j}\right)=e_{j}, \quad j=1, \ldots, n .
$$

Since $\mathbf{T}(f)_{[0, \mu] \cap \Sigma}$ is invertible, uniqueness of $x_{j}, j=1, \ldots, n$ (and thus also uniqueness of $x$ ) follows.

We are now ready to apply the abstract band method; we shall use the notation of Chapter XXXIV in [17]. The original results may be found in [18]-[22] and [40].

Let $\Sigma \subseteq \mathbb{R}$ be an additive subgroup, and let $0<\mu \in \Sigma$. Denote

$$
\begin{gathered}
\mathcal{M}=(A P W)_{\Sigma}^{n \times n}, \mathcal{M}_{1}=\mathcal{M}_{4}^{*}=(A P W)_{\Sigma \cap(\mu, \infty)}^{n \times n}, \\
\mathcal{M}_{2}^{0}=\mathcal{M}_{3}^{0 *}=(A P W)_{\Sigma \cap(0, \mu]}^{n \times n}, \mathcal{M}_{d}=(A P W)_{\{0\}}^{n \times n}\left(\cong \mathbb{C}^{n \times n}\right) .
\end{gathered}
$$

Here the involution $*$ on $(A P W)^{n \times n}$ is defined by

$$
f^{*}(t)=(f(t))^{*}, \quad f \in(A P W)_{\Sigma}^{n \times n}, \quad t \in \mathbb{R},
$$

where for a matrix $M$ we denote its adjoint by $M^{*}$. Clearly,

$$
\mathcal{M}=\mathcal{M}_{1} \dot{+} \mathcal{M}_{2}^{0}+\mathcal{M}_{d}+\mathcal{M}_{3}^{0}+\mathcal{M}_{4} \text {. }
$$

Moreover, $\mathcal{M}$ is an algebra with band structure (8.14) as defined in Chapter XXXIV, Section 1 in [17]. Let $G \in(A P W)_{\Sigma}^{n \times n}$ be positive, i.e., there exists an $\epsilon>0$ such that

$$
G(t) \geq \epsilon I, \quad t \in \mathbb{R}
$$

This implies, in particular, that operator $\mathbf{R}(G)$ of Section 5 is invertible, and combining Theorems 5.1 and 6.3 we obtain that the function $G(t)$ admits the factorizations

$$
\begin{aligned}
G(t)=G_{+}^{*}(t) D_{1}(G) G_{+}(t), & t \in \mathbb{R}, \\
G(t)=G_{-}^{*}(t) D_{2}(G) G_{-}(t), & t \in \mathbb{R},
\end{aligned}
$$

where

$$
\begin{gathered}
G_{+}^{ \pm 1} \in(A P W)_{[0, \infty) \cap \Sigma}^{n \times n}=: \mathcal{M}_{+} ; G_{-}^{ \pm 1} \in(A P W)_{(-\infty, 0] \cap \Sigma}^{n \times n}=: \mathcal{M}_{-} ; \\
D_{1}(G), D_{2}(G) \in \mathcal{M}_{d}, M\left\{G_{+}\right\}=M\left\{G_{-}\right\}=I_{n},
\end{gathered}
$$

are uniquely determined by $G$. (The constant matrices $D_{1}(G), D_{2}(G)$ are both positive definite.) We will call (8.15) (resp. (8.16)) the right (resp. left) spectral factorization of $G$. The existence of the right (or left) spectral factorization for a positive $G$ implies that positivity in the sense above coincides with the positivity 
in the sense of the band method (i.e., $G$ is positive if and only if $G=Q Q^{*}$ with $\left.Q^{ \pm 1} \in(A P W)_{\Sigma}^{n \times n}\right)$.

Proof of Theorem 3.1. We have already established (i) $\Rightarrow$ (ii) $\Leftrightarrow$ (iii) $\Leftrightarrow$ (v) $\Leftrightarrow$ (vi) and (i) $\Rightarrow$ (iv) $\Rightarrow$ (v).

Assume now that (ii) holds, i.e., $\mathbf{T}(f)_{[0, \mu] \cap \Sigma}>0$. By Lemma 8.3 there exist $x \in \mathcal{M}_{2}^{0}+\mathcal{M}_{d}$ and $y \in \mathcal{M}_{3}^{0}+\mathcal{M}_{d}$ such that $f x \in I+\mathcal{M}_{4}+\mathcal{M}_{3}^{0}+\mathcal{M}_{1}$, fy $\in$ $I+\mathcal{M}_{4}+\mathcal{M}_{2}^{0}+\mathcal{M}_{1}$, and $x^{-1} \in \mathcal{M}_{+}, y^{-1} \in \mathcal{M}_{-}, M\{x\}>0$ and $M\{y\}>0$. By Theorem 1.1 in Chapter XXXIV of [17] we obtain that $f$ has a positive extension $h_{0}$ (in fact, a so-called "band extension"), which is given by

$$
h_{0}=x^{*-1} M\{x\} x^{-1} .
$$

By Theorems 1.2 and 1.3 there it follows that $h_{0}$ may also be found via

$$
h_{0}=y^{*-1} M\{y\} y^{-1} .
$$

This shows that (i) holds, and by Lemma 8.3 we have that $x$ and $y$ are given by (3.4). This finishes the proof.

Theorem 8.4. Let $f \in \mathcal{M}_{2}^{0}+\mathcal{M}_{d}+\mathcal{M}_{3}^{0}$ be such that

$$
\mathbf{T}(f)_{[0, \mu] \cap \Sigma}>0 .
$$

Then for any positive extension $h$ in $(A P W)_{\Sigma}^{n \times n}$ of $f$ we have

$$
D_{1}(h) \leq D_{1}\left(h_{0}\right)
$$

where $h_{0}$ is as in Theorem 3.1 and $D_{1}(\cdot)$ is defined by (8.15). Moreover, equality holds in (8.17) if and only if $h=h_{0}$.

An analogous result holds for $D_{2}(\cdot)$ (defined by (8.16)) in place of $D_{1}(\cdot)$.

Proof. Let $\mathcal{R}=\left(L^{\infty}(\mathbb{R})\right)^{n \times n}$. Then $\mathcal{M}$ is "an algebra with band structure (8.14) in the unital $C^{*}$-algebra $\mathcal{R}$," as defined in Section XXXIV.1 in [17]. We need to check that $\mathcal{M}$ with decomposition (8.14) satisfies Axioms (C1) and (C2) in Chapter XXXIV, Section 4 of [17]. Indeed, if $G \in \mathcal{M}$ is positive then its mean $M\{G\}$ is a positive semi-definite matrix, and $M\{G\}$ equals zero if and only if $G \equiv 0$. Thus we may apply Theorem 4.2 in Chapter XXXIV in [17] to obtain the result.

Using Lemma 8.1, and applying Theorem 8.4 for the case $\Sigma=\mathbb{R}$, we see that (8.17) holds also for any positive extension $h \in(A P W)^{n \times n}$ of $f$, with equality only for $h=h_{0}$.

Proof of Theorem 3.2. Suppose that $h \in(A P W)_{\Sigma}^{n \times n}$ is another positive extension of $f$ with $\sigma\left(h^{-1}\right) \subseteq[-\mu, \mu](\cap \Sigma)$. Since $h_{0}$ allows a right spectral factorization and $h$ a left one (in fact, both $h_{0}$ and $h$ allow both factorizations), we obtain from Theorem 1.3 in Chapter XXXIV of [17] that $h_{0}=h$.

Furthermore, note that if $G$ has the right spectral factorization (8.15), then

$$
\log (\operatorname{det} G)=\log \left(\operatorname{det} G_{+}^{*}\right)+\log \operatorname{det} D(G)+\log \left(\operatorname{det} G_{+}\right),
$$

and since $G_{+}^{ \pm 1} \in I+(A P W)_{(0, \infty)}^{n \times n}$ we have by Proposition 2.9 that $M\left\{\log \left(\operatorname{det} G_{+}\right)\right\}$ $=0$. Thus

$$
\Delta(G)=\log \operatorname{det} D_{1}(G) .
$$


But then it follows from (8.17) that

$$
\Delta(h)=\log \operatorname{det} D_{1}(h) \leq \log \operatorname{det} D_{1}\left(h_{0}\right)=\Delta(h)
$$

with equality if and only if $h=h_{0}$ (since for matrices $M \neq N$ with $M \geq N$ we have $\operatorname{det} M>\operatorname{det} N$; in the terminology of Section XXXIV.4 in [17], the function "log det" is strictly $\mathcal{R}$-monotone).

Proof of Theorem 3.3. Let $\mathcal{R}=\left(L^{\infty}(\mathbb{R})\right)^{n \times n}$. Then, as observed before, $\mathcal{M}$ is "an algebra with band structure (8.14) in the unital $C^{*}$-algebra $\mathcal{R}$ ", as defined in Chapter XXXIV of [17]. Next, note that the Axiom (A) in Chapter XXXIV of [17] is satisfied; in other words, if $F \in \mathcal{M}_{+}\left(=(A P W)_{[0, \infty) \cap \Sigma}^{n \times n}\right)$ is such that $\sup _{t \in \mathbb{R}}\|F(t)\|<1$, then $(I-F)^{-1} \in \mathcal{M}_{+}$. Indeed, by Proposition 2.3 (with $f=I-F$ and $\left.\Psi(z)=z^{-1}\right)$ we obtain $(I-F)^{-1} \in(A P W)^{n \times n}$; on the other hand, the series $(I-F)^{-1}=I+F+F^{2}+\cdots$ converges in $(A P)_{[0, \infty) \cap \Sigma}^{n \times n}$; hence by Proposition 2.4 (a) we have $(I-F)^{-1} \in(A P)_{[0, \infty) \cap \Sigma}^{n \times n}$. So $(I-F)^{-1} \in(A P W)^{n \times n} \cap(A P)_{[0, \infty) \cap \Sigma}^{n \times n}=\mathcal{M}_{+}$, as required. Since Axiom (A) in Chapter XXXIV of [17] holds, we may apply Theorem 2.1 of Chapter XXXIV in [17], yielding the present result.

\section{Proofs of the Main Results For the Contraction Extensions Problem}

In this section Theorems $4.1-4.3$ are proved. We keep the notation introduced in Section 4. Throughout this section $\Sigma \subseteq \mathbb{R}$ is an additive subgroup.

We first examine the connections with the Hankel operators defined by (4.1).

Lemma 9.1. Let $f \in(A P)^{m \times n}$. Then

$$
\left\|\mathbf{H}(f)_{\Sigma}\right\|=\|\mathbf{H}(f)\|=\inf _{\tilde{f} \in(A P W)_{(0, \infty)}^{m \times n}} \sup _{t \in \mathbb{R}}\|f(t)+\widetilde{f}(t)\| .
$$

Proof. The second equality in (9.1) follows from Theorem 5.2. The inequality $\left\|\mathbf{H}(f)_{\Sigma}\right\| \leq\|\mathbf{H}(f)\|$ is obvious because $\mathbf{H}(f)_{\Sigma}$ is the restriction of $\mathbf{H}(f)$ to its invariant subspace $(B)_{[0, \infty) \cap \Sigma}^{n \times 1}$.

To prove the remaining inequality $\left\|\mathbf{H}(f)_{\Sigma}\right\| \geq\|\mathbf{H}(f)\|$ we employ a method similar to the proof of Lemma 8.2.

Let $\Omega$ be a set having the cardinality of the set of non-trivial cosets of $\Sigma$ in $\mathbb{R}$, and let $\mathbb{R}=\Sigma \cup \bigcup_{\omega \in \Omega} \Sigma_{\omega}$ be the representation of $\mathbb{R}$ as a disjoint union of cosets of $\Sigma$. Assume first that $\Sigma$ is dense in $\mathbb{R}$.

For every coset $\Sigma_{\omega}(\neq \Sigma)$ select a decreasing sequence of positive elements $\left\{r_{\omega, p}\right\}_{p=1}^{\infty}$ in $\Sigma_{\omega}$ such that $r_{\omega, p}<1 / p$ for all $p$ and all $\omega \in \Omega$. The definition of $\mathbf{H}(f)$ and the multiplication formula (2.1) imply that $\mathbf{H}(f) \operatorname{maps}(B)_{[0, \infty) \cap \Sigma_{\omega}}^{n \times 1}$ into $(B)_{(-\infty, 0] \cap \Sigma_{\omega}}^{m \times 1}$. Now fix $\omega \in \Omega$ and a positive integer $p$, and consider the unitary operators

$$
\Phi_{\omega, p}:(B)_{\left[r_{\omega, p}, \infty\right) \cap \Sigma_{\omega}}^{n \times 1} \rightarrow(B)_{[0, \infty) \cap \Sigma}^{n \times 1}, \quad \Psi_{\omega, p}:(B)_{(-\infty, 0] \cap \Sigma}^{m \times 1} \rightarrow(B)_{\left(-\infty, r_{\omega, p}\right] \cap \Sigma_{\omega}}^{m \times 1}
$$

defined by

$$
\begin{gathered}
\left(\Phi_{\omega, p} g\right)(t)=e^{-i r_{\omega, p} t} g(t), \quad g \in(B)_{\left[r_{\omega, p}, \infty\right) \cap \Sigma_{\omega}}^{n \times 1} ; \\
\left(\Psi_{\omega, p} h\right)(t)=e^{i r_{\omega, p} t} h(t), \quad h \in(B)_{(-\infty, 0] \cap \Sigma}^{m \times 1} .
\end{gathered}
$$


We then have, for every $g \in(B)_{\left[r_{\omega, p}, \infty\right) \cap \Sigma_{\omega}}^{n \times 1}$,

$$
\begin{aligned}
\mathbf{H}(f) g & =\Pi_{(-\infty, 0]}(f g)=\Pi_{(-\infty, 0]}\left(e^{i r_{\omega, p} t} f\left(\Phi_{\omega, p} g\right)\right) \\
& =\Pi_{(-\infty, 0]}\left(e^{i r_{\omega, p} t} \Pi_{(-\infty, 0]}\left(f\left(\Phi_{\omega, p} g\right)\right)\right)=\Pi_{(-\infty, 0]} \Psi_{\omega, p} \mathbf{H}(f)_{\Sigma} \Phi_{\omega, p} g .
\end{aligned}
$$

Let

$$
\Gamma_{p}=([0, \infty) \cap \Sigma) \cup \bigcup_{\omega \in \Omega}\left(\left[r_{\omega, p}, \infty\right) \cap \Sigma_{\omega}\right) .
$$

Define the unitary operator

$$
\Phi_{p}:(B)_{\Gamma_{p}}^{n \times 1} \rightarrow(B)_{[0, \infty) \cap \Sigma}^{n \times 1} \oplus \bigoplus_{\omega \in \Omega}(B)_{[0, \infty) \cap \Sigma}^{n \times 1}
$$

by $I:(B)_{[0, \infty) \cap \Sigma}^{n \times 1} \rightarrow(B)_{[0, \infty) \cap \Sigma}^{n \times 1}$ on the first orthogonal component of

$$
(B)_{\Gamma_{p}}^{n \times 1}=(B)_{[0, \infty) \cap \Sigma}^{n \times 1} \oplus \bigoplus_{\omega \in \Omega}(B)_{\left[r_{\omega, p}, \infty\right) \cap \Sigma_{\omega}}^{n \times 1}
$$

and by $\Phi_{\omega, p}$ on the orthogonal component of $(B)_{\Gamma_{p}}^{n \times 1}$ which is indexed by $\omega$. Analogously (using the unitary operators $\Psi_{\omega, p}$ ) define the unitary operator

$$
\Psi_{p}:(B)_{[0, \infty) \cap \Sigma}^{m \times 1} \oplus \bigoplus_{\omega \in \Omega}(B)_{[0, \infty) \cap \Sigma}^{m \times 1} \rightarrow(B)_{\Delta_{p}}^{m \times 1}
$$

where

$$
\Delta_{p}=((-\infty, 0] \cap \Sigma) \cup \bigcup_{\omega \in \Omega}\left(\left(-\infty, r_{\omega, p}\right] \cap \Sigma_{\omega}\right) .
$$

Equality (9.2) now implies

$$
\mathbf{H}(f) g=\Pi_{(-\infty, 0]} \Psi_{p}\left(\mathbf{H}(f)_{\Sigma} \oplus \bigoplus_{\omega \in \Omega}\left(\mathbf{H}(f)_{\Sigma}\right)\right) \Phi_{p} g
$$

for every $g \in(B)_{\Gamma_{p}}^{n \times 1}$. Consequently,

$$
\left\|\mathbf{H}(f) \mid(B)_{\Gamma_{p}}^{n \times 1}\right\| \leq\left\|\mathbf{H}(f)_{\Sigma}\right\| .
$$

Now observe that $(B)_{\Gamma_{p}}^{n \times 1}(p=1,2, \ldots)$ is an increasing sequence of subspaces in $(B)_{[0, \infty)}^{n \times 1}$ whose union is dense in $(B)_{[0, \infty)}^{n \times 1}$. It follows from (9.3) that $\left\|\mathbf{H}(f)_{\Sigma}\right\| \geq$ $\|\mathbf{H}(f)\|$.

If $\Sigma$ is discrete, then an analogous procedure works. Namely, for every coset $\Sigma_{\omega}(\neq \Sigma)$ let $r_{\omega}$ be the minimal positive element in $\Sigma_{\omega}$. Then the interval $[0, \infty)$ can be represented as the disjoint union $\left.([0, \infty) \cap \Sigma) \cup \bigcup_{\omega \in \Omega}\left[r_{\omega}, \infty\right) \cap \Sigma_{\omega}\right)$. Let also

$$
\Delta=((-\infty, 0] \cap \Sigma) \cup \bigcup_{\omega \in \Omega}\left(\left(-\infty, r_{\omega}\right] \cap \Sigma_{\omega}\right) .
$$

Now, using the unitary operators

$$
\Phi_{\omega}:(B)_{\left[r_{\omega}, \infty\right) \cap \Sigma_{\omega}}^{n \times 1} \rightarrow(B)_{[0, \infty) \cap \Sigma}^{n \times 1}, \quad \Psi_{\omega}:(B)_{(-\infty, 0] \cap \Sigma}^{m \times 1} \rightarrow(B)_{\left(-\infty, r_{\omega}\right] \cap \Sigma_{\omega}}^{m \times 1}
$$

defined by

$$
\begin{gathered}
\left(\Phi_{\omega} g\right)(t)=e^{-i r_{\omega} t} g(t), \quad g \in(B)_{\left[r_{\omega}, \infty\right) \cap \Sigma_{\omega}}^{n \times 1} \\
\left(\Psi_{\omega} h\right)(t)=e^{i r_{\omega} t} h(t), \quad h \in(B)_{(-\infty, 0] \cap \Sigma}^{m \times 1},
\end{gathered}
$$


and applying the above construction given for the case of a dense $\Sigma$, we obtain

$$
\mathbf{H}(f) g=\Pi_{(-\infty, 0]} \Psi\left(\mathbf{H}(f)_{\Sigma} \oplus \bigoplus_{\omega \in \Omega}\left(\mathbf{H}(f)_{\Sigma}\right)\right) \Phi g
$$

for all $g \in(B)_{[0, \infty)}^{n \times 1}$, where $\Phi$ and $\Psi$ are unitary operators.

Hence the inequality $\|\mathbf{H}(f)\| \leq\left\|\mathbf{H}(f)_{\Sigma}\right\|$ follows also for discrete $\Sigma$.

In fact, a result stronger than (9.1) holds (Corollary 9.6 below). We will establish it later, as a byproduct of the proof of Theorem 4.1.

The following lemmas set the stage for application of the band method.

Lemma 9.2. Suppose $f \in(A P W)_{(-\infty, 0] \cap \Sigma}^{m \times n}$ is such that $\|\mathbf{H}(f)\|<1$. Then the equation

$$
\left[\begin{array}{cc}
I_{m} & f \\
f^{*} & I_{n}
\end{array}\right]\left[\begin{array}{c}
x_{12}^{-} \\
x_{22}^{+}
\end{array}\right]=\left[\begin{array}{c}
g_{++} \\
g_{-}
\end{array}\right]
$$

has a solution $x_{12}^{-} \in(A P W)_{\Sigma}^{m \times n}, g_{++} \in(A P W)_{\Sigma}^{m \times n}, x_{22}^{+} \in(A P W)_{\Sigma}^{n \times n}, g_{-} \in$ $(A P W)_{\Sigma}^{n \times n}$ with

$$
\begin{aligned}
\sigma\left(x_{12}^{-}\right) & \subseteq(-\infty, 0], & \sigma\left(x_{22}^{+}\right) & \subseteq[0, \infty) \\
\sigma\left(g_{++}\right) & \subseteq(0, \infty), & \sigma\left(g_{-}-I\right) & \subseteq(-\infty, 0) .
\end{aligned}
$$

The solution is unique and given via

$$
x_{12}^{-}=-\mathbf{H}(f)_{\Sigma}\left(I-\left(\mathbf{H}(f)_{\Sigma}\right)^{*} \mathbf{H}(f)_{\Sigma}\right)^{-1}\left(I_{n}\right), x_{22}^{+}=\left(I-\left(\mathbf{H}(f)_{\Sigma}\right)^{*} \mathbf{H}(f)_{\Sigma}\right)^{-1}\left(I_{n}\right),
$$

where the operators are applied columnwise.

Proof. Let

$$
G=\left[\begin{array}{cc}
I-f f^{*} & f \\
f^{*} & -I
\end{array}\right]
$$

Note the equality

$$
G=\left[\begin{array}{rr}
I & -f \\
0 & I
\end{array}\right]\left[\begin{array}{cc}
I_{m} & 0 \\
0 & -I_{n}
\end{array}\right]\left[\begin{array}{cc}
I & 0 \\
-f^{*} & I
\end{array}\right]
$$

(which can be easily verified), which implies, in particular, that $\operatorname{det} G \equiv(-1)^{n}$ and the signature of the hermitian matrix $G(t), t \in \mathbb{R}$, is constant, with $m$ positive and $n$ negative eigenvalues.

Now let $\widetilde{f}$ be a matrix function in $(A P W)_{(0, \infty)}^{m \times n}$, for which $\sup _{t \in \mathbf{R}}\|f(t)+\widetilde{f}(t)\|<1$ (such an $\tilde{f}$ exists due to condition $\|\mathbf{H}(f)\|<1$ and Lemma 9.1). Then

$$
G=\left[\begin{array}{cc}
I & \tilde{f} \\
0 & I
\end{array}\right] G_{1}\left[\begin{array}{cc}
I & 0 \\
-\widetilde{f}^{*} & -I
\end{array}\right],
$$

where

$$
G_{1}=\left[\begin{array}{cc}
I-(f+\widetilde{f})(f+\widetilde{f})^{*} & -(f+\widetilde{f}) \\
(f+\widetilde{f})^{*} & I
\end{array}\right]
$$


Since

$$
\begin{aligned}
\operatorname{Re} G_{1} & =\left[\begin{array}{cc}
I-(f+\widetilde{f})(f+\widetilde{f})^{*} & 0 \\
0 & I
\end{array}\right] \\
& \geq\left(1-\|f+\widetilde{f}\|^{2}\right) I,
\end{aligned}
$$

the operator $\mathbf{R}\left(G_{1}\right)=\Pi_{(0, \infty)}+\mathbf{M}_{G_{1}} \Pi_{(-\infty, 0]}$ is invertible. Hence, by Theorem 5.1, the matrix $G_{1}$ has a $W$-canonical factorization. Since the latter property is invariant under multiplication by invertible in $(A P W)_{[0, \infty)}^{(m+n) \times(m+n)}$ matrices from the left and invertible in $(A P W)_{(-\infty, 0]}^{(m+n) \times(m+n)}$ matrices from the right, there exists a $W$-canonical factorization of $G$ as well. It can be taken in the form given by Theorem 6.3. In other words, there exist

$\alpha \in(A P W)_{[0, \infty) \cap \Sigma}^{m \times m}, \beta \in(A P W)_{[0, \infty) \cap \Sigma}^{m \times n}, \gamma \in(A P W)_{[0, \infty) \cap \Sigma}^{n \times m}, \delta \in(A P W)_{[0, \infty) \cap \Sigma}^{n \times n}$, such that

$$
\left[\begin{array}{cc}
\alpha & \beta \\
\gamma & \delta
\end{array}\right]^{ \pm 1} \in(A P W)_{[0, \infty) \cap \Sigma}^{(m+n) \times(m+n)}
$$

and

$$
G(t)=A(t) J(A(t))^{*}, t \in \mathbb{R}
$$

where $A=\left[\begin{array}{ll}\alpha & \beta \\ \gamma & \delta\end{array}\right]$ and $J=\left(I_{m}\right) \oplus\left(-I_{n}\right)$. Moreover, since $\operatorname{det} G \equiv(-1)^{n}$, we can take $A$ so that

$$
\operatorname{det} A \equiv 1
$$

Indeed, it follows from (9.7) and (9.8) that $|\operatorname{det} A(t)|=1$ for $t \in \mathbb{R}$. Proposition 2.9 implies that $(\operatorname{det} A(t))^{ \pm \frac{1}{m+n}} \in(A P W)_{[0, \infty) \cap \Sigma}$. So we can replace $A(t)$ by ( $\operatorname{det} A(t))^{-\frac{1}{m+n}} A(t)$ to ensure the property (9.9).

We obtain from (9.8) that

$$
\left.\begin{array}{l}
I_{m}-f f^{*}=\alpha \alpha^{*}-\beta \beta^{*} \\
f=\alpha \gamma^{*}-\beta \delta^{*},-I_{n}=\gamma \gamma^{*}-\delta \delta^{*}
\end{array}\right\} .
$$

Further, note the equality

$$
\left[\begin{array}{cc}
I_{m} & f \\
f^{*} & -I_{n}+f^{*} f
\end{array}\right]=G^{-1}=A^{*-1} J A^{-1} .
$$

Hence, if we denote

$$
A^{-1}=\left[\begin{array}{ll}
p & q \\
r & s
\end{array}\right]
$$

we obtain that

$$
\left.\begin{array}{l}
I_{n}=p^{*} p-r^{*} r, f=p^{*} q-r^{*} s \\
-I_{n}+f^{*} f=q^{*} q-s^{*} s
\end{array}\right\} .
$$

Now let

$$
y_{+}=-\gamma q_{0}-\delta s_{0}, y_{-}=p^{*} q_{0}-r^{*} s_{0},
$$


where $q_{0}=M\{q\}$ and $s_{0}=M\{s\}$. Since $\|\mathbf{H}(f)\|<1$, we have

$$
\left[\begin{array}{cc}
I & \mathbf{H}(f) \\
(\mathbf{H}(f))^{*} & I
\end{array}\right]>0 .
$$

So, in particular, for all $0 \neq v \in \mathbb{C}^{n}$,

$$
\left\langle\left[\begin{array}{cc}
I & \mathbf{H}(f) \\
\mathbf{H}(f)^{*} & I
\end{array}\right]\left[\begin{array}{l}
y_{-} \\
y_{+}
\end{array}\right] v,\left[\begin{array}{l}
y_{-} \\
y_{+}
\end{array}\right] v\right\rangle>0 .
$$

To see that indeed the strict inequality holds in (9.14), note that

$$
\left(y_{+} v\right)_{0}=M\left\{y_{+} v\right\}=M\left\{-\gamma q_{0} v-\delta s_{0} v\right\}=M\{-\gamma q v-\delta s v\},
$$

which is equal to $-v$ in view of the multiplicativity of $M\{\cdot\}$ on $(A P W)_{[0, \infty)}$; hence $\left[\begin{array}{l}y_{-} \\ y_{+}\end{array}\right] v \neq 0$ as long as $v \neq 0$. Now make a calculation:

$$
\begin{aligned}
f y_{+} & =\left(p^{*} q-r^{*} s\right)\left(-\gamma q_{0}-\delta s_{0}\right) \quad \text { by }(9.12),(9.13) \\
& =\left(-p^{*} q \gamma+r^{*} s \gamma\right) q_{0}+\left(-p q \delta+r^{*} s \delta\right) s_{0} \\
& =\left(-p^{*}(I-p \alpha)+r^{*}(-r \alpha)\right) q_{0}+\left(-p^{*}(-p \beta)+r^{*}(I-r \beta)\right) s_{0} \quad \text { by }(9.11) \\
& =\left(-p^{*}+\alpha\right) q_{0}+\left(r^{*}+\beta\right) s_{0} \quad \text { by }(9.12) .
\end{aligned}
$$

So, since $\alpha q+\beta s \equiv 0$ (in particular, $M\left\{\alpha q_{0}+\beta s_{0}\right\}=M\{\alpha q+\beta s\}=0$ ), we obtain

$$
\mathbf{H}(f) y_{+}=-p^{*} q_{0}+r^{*} s_{0} .
$$

Similarly,

$$
\begin{aligned}
f^{*} y_{-} & =\left(\gamma \alpha^{*}-\delta \beta^{*}\right)\left(p^{*} q_{0}-r^{*} s_{0}\right) \quad \text { by }(9.10),(9.13) \\
& =\left(\gamma \alpha^{*} p^{*}-\delta \beta^{*} p^{*}\right) q_{0}-\left(\gamma \alpha^{*} r^{*}-\delta \beta^{*} r^{*}\right) s_{0} \\
& =\left(\gamma\left(I-\gamma^{*} q^{*}\right)-\delta\left(-\delta^{*} q^{*}\right)\right) q_{0}-\left(\gamma\left(-\gamma^{*} s^{*}\right)-\delta\left(I-\delta^{*} s^{*}\right)\right) s_{0} \quad \text { by }(9.11) \\
& =\gamma q_{0}+q^{*} q_{0}-s^{*} s_{0}+\delta s_{0} \quad \text { by }(9.10) .
\end{aligned}
$$

Thus

$$
\mathbf{H}(f)^{*} y_{-}=\Pi_{[0, \infty)} f^{*} y_{-}=\gamma q_{0}+q_{0}^{*} q_{0}-s_{0}^{*} s_{0}+\delta s_{0} .
$$

So the left-hand side of (9.14) equals

$$
\begin{aligned}
& v^{*} M\left\{y_{-}^{*}\left(y_{-}+\mathbf{H}(f) y_{+}\right)+y_{+}^{*}\left(y_{+}+\mathbf{H}(f)^{*} y_{-}\right)\right\} v \\
& =v^{*} M\left\{y_{-}^{*}\left(p^{*} q_{0}-r^{*} s_{0}-p^{*} q_{0}+r^{*} s_{0}\right)\right. \\
& \left.\quad+y_{+}^{*}\left(-\gamma q_{0}-\delta s_{0}+\gamma q_{0}+q_{0}^{*} q_{0}-s_{0}^{*} s_{0}+\delta s_{0}\right)\right\} v \\
& =0+v^{*} M\left\{y_{+}^{*}\right\}\left(q_{0}^{*} q_{0}-s_{0}^{*} s_{0}\right) v=v^{*}\left(s_{0}^{*} s_{0}-q_{0}^{*} q_{0}\right) v,
\end{aligned}
$$

where in the last step we use the equalities $M\left\{y_{+}\right\}=-M\{\gamma q+\delta s\}=-I$. But then we get that

$$
s_{0}^{*} s_{0}-q_{0}^{*} q_{0}>0
$$


Now let

$$
\begin{aligned}
g_{++} & =\left(\alpha q_{0}+\beta s_{0}\right)\left(-s_{0}^{*} s_{0}+q_{0}^{*} q_{0}\right)^{-1}, \\
g_{-} & =\left(q^{*} q_{0}-s^{*} s_{0}\right)\left(-s_{0}^{*} s_{0}+q_{0}^{*} q_{0}\right)^{-1}, \\
x_{12}^{-} & =\left(p^{*} q_{0}-r^{*} s_{0}\right)\left(-s_{0}^{*} s_{0}+q_{0}^{*} q_{0}\right)^{-1}, \\
x_{22}^{+} & =\left(-\gamma q_{0}-\delta s_{0}\right)\left(-s_{0}^{*} s_{0}+q_{0}^{*} q_{0}\right)^{-1} .
\end{aligned}
$$

Using the formulas for $f$ from (9.10) and (9.12), we verify that these functions indeed provide a solution to (9.4). Clearly, the conditions (9.5) are satisfied as well. As for the condition (9.6), observe that

$$
M\left\{g_{++}\right\}=\left(\alpha_{0} q_{0}+\beta_{0} s_{0}\right)\left(-s_{0}^{*} s_{0}+q_{0}^{*} q_{0}\right)^{-1}=0
$$

because $\alpha q+\beta s \equiv 0$, and $M\left\{g_{-}\right\}=I$.

For the uniqueness, observe that

$$
\left[\begin{array}{cc}
\Pi_{(-\infty, 0] \cap \Sigma} & 0 \\
0 & \Pi_{[0, \infty) \cap \Sigma}
\end{array}\right]\left[\begin{array}{cc}
I_{m} & f \\
f^{*} & I_{n}
\end{array}\right]\left[\begin{array}{c}
x_{12}^{-} \\
x_{22}^{+}
\end{array}\right]=\left[\begin{array}{c}
\Pi_{(-\infty, 0] \cap \Sigma}\left(g_{++}\right) \\
\Pi_{[0, \infty) \cap \Sigma}\left(g_{-}\right)
\end{array}\right]=\left[\begin{array}{c}
0 \\
I_{n}
\end{array}\right] .
$$

So, using $\left\|\mathbf{H}(f)_{\Sigma}\right\|=\|\mathbf{H}(f)\|<1$, we get

$$
\begin{aligned}
{\left[\begin{array}{c}
x_{12}^{-} \\
x_{22}^{+}
\end{array}\right] } & =\left[\begin{array}{cc}
I & \mathbf{H}(f)_{\Sigma} \\
\mathbf{H}(f)_{\Sigma}^{*} & I
\end{array}\right]^{-1}\left[\begin{array}{c}
0 \\
I_{n}
\end{array}\right] \\
& =\left[\begin{array}{c}
-\mathbf{H}(f)_{\Sigma}\left(I-\left(\mathbf{H}(f)_{\Sigma}\right)^{*} \mathbf{H}(f)_{\Sigma}\right)^{-1}\left(I_{n}\right) \\
\left(I-\left(\mathbf{H}(f)_{\Sigma}\right)^{*} \mathbf{H}(f)_{\Sigma}\right)^{-1}\left(I_{n}\right)
\end{array}\right]
\end{aligned}
$$

Remark 9.3. Invertibility of the matrices

$$
\left[\begin{array}{cc}
I_{m} & \tilde{f} \\
0 & I_{n}
\end{array}\right] \text { and }\left[\begin{array}{cc}
I_{m} & 0 \\
-\widetilde{f}^{*} & -I_{n}
\end{array}\right]
$$

in $(A P W)_{[0, \infty)}^{(m+n) \times(m+n)}$ and $(A P W)_{(-\infty, 0]}^{(m+n) \times(m+n)}$, respectively, is guaranteed by the condition $\tilde{f} \in(A P W)_{[0, \infty)}^{m \times n}$. Therefore, $G$ admits a $W$-canonical factorization whenever

$$
\|\widetilde{\mathbf{H}}(f)\|=\inf _{\tilde{f} \in(A P W)_{[0, \infty)}^{m \times n}} \sup _{t \in \mathbb{R}}\|f(t)+\widetilde{f}(t)\|<1 .
$$

The latter condition is less restrictive than $\|\mathbf{H}(f)\|<1$. This observation will be used later in Section 10.

Additional properties of the function $x_{22}^{+}$are given in the next lemma.

Lemma 9.4. Let $x_{22}^{+} \in(A P W)_{[0, \infty) \cap \Sigma}^{n \times n}$ be the matrix function constructed in Lemma 9.2. Then $x_{22}^{+}$has an inverse in $(A P W)^{n \times n}$, and $\sigma\left(\left(x_{22}^{+}\right)^{-1}\right) \subseteq[0, \infty) \cap \Sigma$.

Proof. First observe that $\delta \delta^{*} \geq I+\gamma \gamma^{*} \geq I$ by (9.10), and therefore $\delta^{-1} \in$ $(A P W)^{n \times n}$ (indeed, $|\operatorname{det} \delta(t)| \geq 1$ for all $t \in \mathbb{R}$ ). Now write

$$
x_{22}^{+}=-\delta\left(\delta^{-1} \gamma q_{0} s_{0}^{-1}+I\right) s_{0}\left(-s_{0}^{*} s_{0}+q_{0}^{*} q_{0}\right)^{-1},
$$

and observe that $s_{0}$ is invertible in view of (9.16), while

$$
\delta^{-1} \gamma \gamma^{*} \delta^{*-1}=\delta^{-1}\left(\delta \delta^{*}-I\right) \delta^{*-1}=I-\delta^{-1} \delta^{*-1}<I,
$$


and similarly (in view of (9.16)),

$$
s_{0}^{*-1} q_{0}^{*} q_{0} s_{0}^{-1}<I .
$$

Therefore, there exists $\epsilon>0$ such that

$$
\left\|\delta(t)^{-1} \gamma(t) q_{0} s_{0}^{-1}\right\|<1-\epsilon
$$

for all $t \in \mathbb{R}$, and by Proposition 2.3 (with $\Psi(z)=z^{-1}$ )

$$
\left(I+\delta^{-1} \gamma q_{0} s_{0}^{-1}\right)^{-1} \in(A P W)^{n \times n} .
$$

Hence $x_{22}^{+}$is also invertible in $(A P W)^{n \times n}$.

To prove that $x_{22}^{+}$is invertible in $(A P W)_{[0, \infty) \cap \Sigma}^{n \times n}$ we use Theorem 7.2. Consider $u f \in(A P W)_{(0 \infty, 0] \cap \Sigma}^{m \times n}$, where $0 \leq u \leq 1$. When $u=0$, we obtain from (9.4) and (9.6) that $x_{22}^{+} \equiv I$, and therefore trivially $x_{22}^{+}$is invertible in $(A P W)_{[0, \infty)}^{n \times n}$. By Theorem $6.2 x_{22}^{+}$is a continuous function of $u$, and an application of Theorem 7.2 shows that $\sigma\left(\left(x_{22}^{+}\right)^{-1}\right) \subseteq[0, \infty) \cap \Sigma$, as claimed.

Analogously to Lemmas 9.2 and 9.4 one can prove the following.

Lemma 9.5. Suppose $f \in(A P W)_{(-\infty, 0] \cap \Sigma}^{m \times n}$ is such that $\|\mathbf{H}(f)\|<1$. Then the equation

$$
\left[\begin{array}{cc}
I_{m} & f \\
f^{*} & I_{n}
\end{array}\right]\left[\begin{array}{c}
y_{11}^{-} \\
y_{21}^{+}
\end{array}\right]=\left[\begin{array}{c}
h_{+} \\
h_{--}
\end{array}\right]
$$

has a solution $y_{11}^{-}, h_{+} \in(A P W)_{\Sigma}^{m \times m}, y_{21}^{+}, h_{--} \in(A P W)_{\Sigma}^{n \times m}$ with

$$
\sigma\left(y_{11}^{-}\right) \subseteq(-\infty, 0], \sigma\left(y_{21}^{+}\right) \subseteq[0, \infty), \sigma\left(h_{+}-I\right) \subseteq(0, \infty), \sigma\left(h_{--}\right) \subseteq(-\infty, 0) .
$$

The solution is unique, and given via

$$
y_{11}^{-}=\left(I-\mathbf{H}(f)_{\Sigma}\left(\mathbf{H}(f)_{\Sigma}\right)^{*}\right)^{-1}\left(I_{m}\right), y_{21}^{+}=-\mathbf{H}(f)_{\Sigma}\left(I-\mathbf{H}(f)_{\Sigma}\left(\mathbf{H}(f)_{\Sigma}\right)^{*}\right)^{-1}\left(I_{m}\right),
$$

where the operators are applied columnwise.

Furthermore, $y_{11}^{-}$has an inverse in $(A P W)^{m \times m}$, and moreover, $\sigma\left(\left(y_{11}^{-}\right)^{-1}\right) \subseteq$ $(-\infty, 0] \cap \Sigma$.

We now set up the band method application. Let

$$
\begin{aligned}
\mathcal{M}= & (A P W)_{\Sigma}^{(n+m) \times(n+m)}, \\
\mathcal{M}_{d}= & \left\{\left[\begin{array}{cc}
c_{1} & 0 \\
0 & c_{2}
\end{array}\right]: c_{1} \in \mathbb{C}^{m \times m}, c_{2} \in \mathbb{C}^{n \times n}\right\} \\
\mathcal{M}_{1}= & \left\{\left[\begin{array}{cc}
0 & g(t) \\
0 & 0
\end{array}\right]: g \in(A P W)_{(0, \infty) \cap \Sigma}^{m \times n}\right\}=\mathcal{M}_{4}^{*}, \\
\mathcal{M}_{2}^{0}= & \left\{\left[\begin{array}{cc}
g_{1}(t) & g_{3}(t) \\
0 & g_{2}(t)
\end{array}\right]: g_{1} \in(A P W)_{(0, \infty) \cap \Sigma}^{m \times m},\right. \\
& \left.g_{2} \in(A P W)_{(0, \infty) \cap \Sigma}^{n \times n}, g_{3} \in(A P W)_{(-\infty, 0] \cap \Sigma}^{m \times n}\right\}=\mathcal{M}_{3}^{0^{*}},
\end{aligned}
$$


where the involution $*$ is given via

$$
\left[\begin{array}{ll}
a(t) & b(t) \\
c(t) & d(t)
\end{array}\right]^{*}=\left[\begin{array}{ll}
a(t)^{*} & c(t)^{*} \\
b(t)^{*} & d(t)^{*}
\end{array}\right]
$$

Clearly,

$$
\mathcal{M}=\mathcal{M}_{1}+\mathcal{M}_{2}^{0}+\mathcal{M}_{d}+\mathcal{M}_{3}^{0}+\mathcal{M}_{4}
$$

and $\mathcal{M}$ is an algebra with band structure (9.17) as defined in Chapter XXXIV, Section 1 in [17]. When, for some $\epsilon>0$,

$$
G(t)=\left[\begin{array}{ll}
G_{11}(t) & G_{12}(t) \\
G_{12}^{*}(t) & G_{22}(t)
\end{array}\right] \geq \epsilon I_{n+m}, \quad t \in \mathbb{R},
$$

we may factor $G(t)$ as

$$
\begin{aligned}
& G(t)=H_{+}^{*}(t) \widetilde{D}_{1}(G) H_{+}(t), \quad t \in \mathbb{R}, \\
& G(t)=H_{-}^{*}(t) \widetilde{D}_{2}(G) H_{-}(t), \quad t \in \mathbb{R},
\end{aligned}
$$

where

$$
\begin{gathered}
H_{+}^{ \pm 1} \in \mathcal{M}_{+}=\mathcal{M}_{1}+\mathcal{M}_{2}^{0}+\mathcal{M}_{d}, \\
H_{-}^{ \pm 1} \in \mathcal{M}_{-}=\mathcal{M}_{+}^{*}, \widetilde{D}_{i}(G) \in \mathcal{M}_{d}(i=1,2),
\end{gathered}
$$

and

$$
M\left\{H_{+}\right\}=M\left\{H_{-}\right\}=I_{n+m} .
$$

One may show this by using Schur complements, and the factorization results in Section 8 (see (8.15), (8.16)). In particular,

$$
\begin{aligned}
& \widetilde{D}_{1}(G)=\left[\begin{array}{cc}
D_{1}\left(G_{11}\right) & 0 \\
0 & D_{1}\left(G_{22}-G_{12}^{*} G_{11}^{-1} G_{12}\right)
\end{array}\right], \\
& \widetilde{D}_{2}(G)=\left[\begin{array}{cc}
D_{2}\left(G_{11}-G_{12} G_{22}^{-1} G_{12}^{*}\right) & 0 \\
0 & D_{2}\left(G_{22}\right)
\end{array}\right],
\end{aligned}
$$

where $D_{1}$ and $D_{2}$ are defined via (8.15) and (8.16) respectively. Note that when $G_{11}(t) \equiv I$ and $G_{22}(t) \equiv I$, we have that $G(t)$ is positive if and only if there is a $\rho$ such that $\left\|G_{12}(t)\right\| \leq \rho<1$ for all $t \in \mathbb{R}$.

Proof of Theorem 4.1. Using Lemma 9.1, we get (i) $\Leftrightarrow$ (ii) $\Leftrightarrow$ (iv) $\Leftarrow$ (iii).

Assume now that (ii) holds, i.e., $\|\mathbf{H}(f)\|<1$. Introduce $\widehat{\alpha}, \widehat{\beta}, \widehat{\gamma}, \widehat{\delta}$ as in the statement of the theorem, and put

$$
k=\left[\begin{array}{cc}
I & f \\
f^{*} & I
\end{array}\right], x=\left[\begin{array}{cc}
I & -\widehat{\beta} \\
0 & \widehat{\delta}
\end{array}\right], y=\left[\begin{array}{cc}
\widehat{\alpha} & 0 \\
-\widehat{\gamma} & I
\end{array}\right] .
$$

Then, by Lemmas 9.2, 9.4 and 9.5, we get that $k x \in I+\mathcal{M}_{4}+\mathcal{M}_{3}^{0}+\mathcal{M}_{1}, k y \in$ $I+\mathcal{M}_{4}+\mathcal{M}_{2}^{0}+\mathcal{M}_{1}, x^{-1} \in \mathcal{M}_{+}, y^{-1} \in \mathcal{M}_{-}, M\{x\}>0$ and $M\{y\}>0$. By 
Theorems 1.1, 1.2 and 1.3 in Chapter XXXIV of [17] we obtain that $k$ has a positive extension given by

$$
\begin{gathered}
h_{0}=x^{*-1} M\{x\} x^{-1}=y^{*-1} M\{y\} y^{-1} \\
=\left[\begin{array}{cc}
I & \beta \delta^{-1} \\
\left(\beta \delta^{-1}\right)^{*} & I
\end{array}\right]=\left[\begin{array}{cc}
I & \left(\gamma \alpha^{-1}\right)^{*} \\
\gamma \alpha^{-1} & I
\end{array}\right] .
\end{gathered}
$$

Positivity of $h_{0}$ means that

$$
\left\|\beta(t) \delta(t)^{-1}\right\|=\left\|\alpha^{*-1}(t) \gamma^{*}(t)\right\| \leq \rho<1, \quad t \in \mathbb{R},
$$

for some $\rho$. But then $\beta \delta^{-1}=\alpha^{*-1} \gamma^{*}$ is a strictly contractive extension of $f$, proving (iii).

Corollary 9.6. Let $\Sigma$ be an additive subgroup of $\mathbb{R}$. Then, for any $f \in(A P)_{\Sigma}^{m \times n}$,

$$
\begin{aligned}
& \left\|\mathbf{H}(f)_{\Sigma}\right\|=\|\mathbf{H}(f)\| \\
& =\inf _{\tilde{f} \in(A P W)_{(0, \infty)}^{m \times n}} \sup _{t \in \mathbb{R}}\|f(t)+\widetilde{f}(t)\|=\inf _{\tilde{f} \in(A P W)_{(0, \infty) \cap \Sigma}^{m \times n}} \sup _{t \in \mathbb{R}}\|f(t)+\widetilde{f}(t)\| .
\end{aligned}
$$

Proof. The first two equalities in (9.22) follow from Lemma 9.1. Since the inequality

$$
\inf _{\tilde{f} \in(A P W)_{(0, \infty)}^{m \times n}} \sup _{t \in \mathbb{R}}\|f(t)+\widetilde{f}(t)\| \leq \inf _{\tilde{f} \in(A P W)_{(0, \infty) \cap \Sigma}^{m \times n}} \sup _{t \in \mathbb{R}}\|f(t)+\widetilde{f}(t)\|
$$

is obvious, it remains only to show that

$$
\inf _{\tilde{f} \in(A P W)_{(0, \infty)}^{m \times n}} \sup _{t \in \mathbb{R}}\|f(t)+\widetilde{f}(t)\| \geq \inf _{\tilde{f} \in(A P W)_{(0, \infty) \cap \Sigma}^{m \times n}} \sup _{t \in \mathbb{R}}\|f(t)+\widetilde{f}(t)\| .
$$

Due to the density of $(A P W)_{\Sigma}$ in $(A P)_{\Sigma}$, it suffices to consider the case of $f \in$ $(A P W)_{\Sigma}^{m \times n}$. Of course, we may then suppose that $\sigma(f) \subseteq(-\infty, 0]$.

Suppose first that

$$
\inf _{\tilde{f} \in(A P W)_{(0, \infty)}^{m \times n}} \sup _{t \in \mathbb{R}}\|f(t)+\widetilde{f}(t)\|<1 .
$$

For such an $f$, statement (i) of Theorem 4.1 holds. Introduce $g_{0}$ by formula (4.4), and put $\widetilde{f}=g_{0}-f$. According to Theorem 4.1,

$$
\widetilde{f} \in(A P W)_{(0, \infty) \cap \Sigma}^{m \times n} \text { and } \sup _{t \in \mathbb{R}}\|f(t)+\widetilde{f}(t)\|=\left\|g_{0}\right\|<1 .
$$

Hence,

$$
\inf _{\tilde{f} \in(A P W)_{(0, \infty) \cap \Sigma}^{m \times n}} \sup _{t \in \mathbb{R}}\|f(t)+\widetilde{f}(t)\|<1 .
$$

Considering scalar multiples of $f$ instead of $f$, we obtain (9.23).

Theorem 9.7. Let $f \in(A P W)_{(-\infty, 0] \cap \Sigma}^{m \times n}$ be given, where $\Sigma$ is an additive subgroup. Suppose that $\|\mathbf{H}(f)\|<1$. Then for any strictly contractive extension $g$ in $(A P W)^{n \times n}$ of $f$ we have

$$
D_{1}\left(I-g^{*} g\right) \leq D_{1}\left(I-g_{0}^{*} g_{0}\right),
$$

where $g_{0}$ is as in Theorem 4.1 and $D_{1}($.$) is defined by (8.15). Moreover, equality$ holds in (9.24) if and only if $g=g_{0}$.

Analogous results hold for $D_{2}\left(I-g g^{*}\right)$, where $D_{2}$ is defined by (8.16). 
Proof. Let $\mathcal{R}=\left(L^{\infty}(\mathbb{R})\right)^{(n+m) \times(n+m)}$. Then $\mathcal{M}$ is "an algebra with band structure (9.17) in the unital $C^{*}$-algebra $\mathcal{R}$ ", as defined in Section XXXIV.1 in [17]. It is straightforward to check that the decomposition (9.17) satisfies Axioms (C1) and (C2) in Chapter XXXIV, Section 4 of [17]. Applying now Theorem 4.2 in Chapter XXXIV in [17] and using observations (9.20) and (9.21), we obtain the result for $g \in(A P W)_{\Sigma}^{m \times n}$. When we take $\Sigma=\mathbb{R}$, we get the result also for $g \in(A P W)^{m \times n}$.

Proof of Theorem 4.2. Note that from the proof of Theorem 4.1 we get that

$$
\left[\begin{array}{cc}
I & g_{0} \\
g_{0}^{*} & I
\end{array}\right]^{-1}=\left[\begin{array}{cc}
* & g_{0}\left(I-g_{0}^{*} g_{0}\right)^{-1} \\
* & *
\end{array}\right]^{-1} \in \mathcal{M}_{2}^{0}+\mathcal{M}_{d}+\mathcal{M}_{3}^{0} .
$$

Thus $g_{0}\left(I-g_{0}^{*} g_{0}\right)^{-1} \in(A P W)_{(-\infty, 0] \cap \Sigma}^{m \times n}$. Moreover, by Theorem 1.3 in Chapter XXXIV of [17], we get that for any strictly contractive extension $g$ of $f$ with

$$
\left[\begin{array}{cc}
I & g \\
g^{*} & I
\end{array}\right]^{-1} \in \mathcal{M}_{2}^{0}+\mathcal{M}_{d}+\mathcal{M}_{3}^{0}
$$

we have $g=g_{0}$. But then the first statement follows.

For the second statement, apply reasoning similar to the second part of the proof of Theorem 3.2.

Proof of Theorem 4.3. We take the same setup as in the proof of Theorem 9.7. Similarly as in the proof of Theorem 3.3 we may check that Axiom (A) in Chapter XXXIV of [17] is satisfied. By Theorem 2.1 of Chapter XXXIV in [17], we get that all positive extensions of

$$
\left[\begin{array}{cc}
I & f \\
f^{*} & I
\end{array}\right]
$$

are given (in a one-to-one correspondence) by

$$
\begin{aligned}
& \widehat{T}\left(\left[\begin{array}{cc}
0 & -g \\
0 & 0
\end{array}\right]\right)=\left(\left[\begin{array}{cc}
I & -\beta \\
0 & \delta
\end{array}\right]+\left[\begin{array}{cc}
\alpha & 0 \\
-\gamma & I
\end{array}\right]\left[\begin{array}{cc}
0 & -g \\
0 & 0
\end{array}\right]\right)^{*-1} \\
& \cdot\left[\begin{array}{cc}
I & 0 \\
0 & I-g^{*} g
\end{array}\right]\left(\left[\begin{array}{cc}
I & -\beta \\
0 & \delta
\end{array}\right]+\left[\begin{array}{cc}
\alpha & 0 \\
-\gamma & I
\end{array}\right]\left[\begin{array}{cc}
0 & -g \\
0 & 0
\end{array}\right]\right)^{-1}=\left[\begin{array}{cc}
I & T(g) \\
T(g)^{*} & I
\end{array}\right],
\end{aligned}
$$

where $g \in(C A P W)_{(0, \infty) \cap \Sigma}^{m \times n}$. It should be noted that the $2 \times 2$ entry of the product indeed has to equal $I$, since this product is a positive extension of (9.25). This proves the result.

\section{Point-Excluding Variations}

The Carathéodory-Toeplitz and Nehari problems allow the following variations:

C-T II: Let $\mu \in(0, \infty)$ and $f \in(A P W)^{n \times n}$ be such that $\sigma(f) \subseteq(-\mu, \mu)$. What is the condition on $f$ such that there exist an $h \in(A P W)^{n \times n}$ for which:

(1) $h$ is positive;

(2) $f_{\lambda}=h_{\lambda}, \lambda \in(-\mu, \mu)$ ?

N II: Given is an $f \in(A P W)_{(-\infty, 0)}^{m \times n}$. When does there exist an $h \in(A P W)^{m \times n}$ such that

(1) $h$ is strictly contractive;

(2) $f_{\lambda}=h_{\lambda}, \quad \lambda<0$ ? 
The difference from the $\mathbf{C}-\mathbf{T}$ and $\mathbf{N}$ problems is that now $f_{\mu}$ and $f_{0}$ are not prescribed, respectively. For that reason we will call C-T II the point-excluding Carathéodory-Toeplitz problem and N II the point-excluding Nehari problem. For the periodic case, the problems with or without the border point can be reduced to one another (replace $\mu$ by $\mu-1$, for instance), but in this setting one has to treat these variations separately. As it turns out, C-T II and N II have similar answers (with similar proofs) as $\mathbf{C}-\mathbf{T}$ and $\mathbf{N}$, respectively. Rather than giving the full statements with full proofs, we just outline the differences in the statements and proofs as compared with the ones for $\mathbf{C}-\mathbf{T}$ and $\mathbf{N}$.

For C-T II one obtains valid theorems when in Theorems 3.1, 3.2, 3.3 and 8.4 the following changes are made:

(1) replace $f \in(A P W)_{[-\mu, \mu] \cap \Sigma}^{n \times n}$ by $f \in(A P W)_{(-\mu, \mu) \cap \Sigma}^{n \times n}$.

(2) replace the Toeplitz operators by

$$
\widetilde{\mathbf{T}}(f)_{[0, \mu) \cap \Sigma}:(B)_{[0, \mu) \cap \Sigma}^{n \times 1} \rightarrow(B)_{[0, \mu) \cap \Sigma}^{n \times 1}, \quad g \mapsto \prod_{[0, \mu)}(f g), g \in(B)_{[0, \mu) \cap \Sigma}^{n \times 1},
$$

and

$$
\begin{aligned}
& \widetilde{\mathbf{T}}(f)_{(-\mu, 0] \cap \Sigma}:(B)_{(-\mu, 0] \cap \Sigma}^{n \times 1} \rightarrow(B)_{(-\mu, 0] \cap \Sigma}^{n \times 1}, \quad g \mapsto \Pi_{(-\mu, 0]}(f g), g \in(B)_{(-\mu, 0] \cap \Sigma}^{n \times 1} . \\
& \text { (3) } x \in(A P W)_{[0, \mu) \cap \Sigma}^{n \times n}, y \in(A P W)_{[0, \mu) \cap \Sigma}^{n \times n} . \\
& \text { (4) replace }(3.5) \text { by } \sigma\left(h_{0}^{-1}\right) \subseteq(-\mu, \mu) . \\
& \text { (5) replace }(C A P W)_{(\mu, \infty) \cap \Sigma}^{n \times n} \text { by }(C A P W)_{[\mu, \infty) \cap \Sigma}^{n \times n} .
\end{aligned}
$$

In several other places minor changes need to be made (e.g., replace $[-\mu, \mu]$ by $(-\mu, \mu)$, etc.). These are now straightforward.

In order to prove these new results the following observations are needed.

(A) Positivity of $\widetilde{\mathbf{T}}(f)_{[0, \mu) \cap \Sigma}$ implies that $\left[\begin{array}{cc}f(t) & e^{-i \mu t} I_{n} \\ e^{i \mu t} I_{n} & 0\end{array}\right]$ admits a $W$-canonical factorization as in (8.4).

(B) The choice $c_{1}=\gamma_{0}^{*}, c_{2}=-\delta_{0}^{*}$ in (8.7) will result in $M\left\{x_{1}^{-}\right\}=0, M\left\{x_{2}^{-}\right\}=I$.

Subsequently, (8.10) will result in the conclusion that $M\left\{x_{1}^{+*}\right\}<0$. By choosing $\widehat{c_{1}}=-c_{1}=-\gamma_{0}^{*}$ and $\widehat{c_{2}}=-c_{2}=\delta_{0}^{*}$, and setting $x=\gamma \widehat{c_{1}}+\delta \widehat{c_{2}}$, we get the desired $x$ for the point-excluding variation of Lemma 8.3.

To check that (B) results in valid statements requires purely algebraic manipulations. In its turn, (A) follows from the fact that $\widetilde{\mathbf{T}}(f)_{[0, \mu) \cap \Sigma}>0$ if and only if $\widetilde{\mathbf{T}}(f)_{[0, \mu)}>0$ (established similarly to Lemma 8.1) and Lemma 10.1 below.

Lemma 10.1. Let the operator $\widetilde{\mathbf{T}}(f)_{[0, \mu)}$ be invertible. Then the matrix function

$$
\left[\begin{array}{cc}
f(t) & e^{-i \mu t} I_{n} \\
e^{i \mu t} I_{n} & 0
\end{array}\right]
$$

admits a factorization (5.3) satisfying (5.5).

Proof. Due to Theorem 5.1, it suffices to prove the invertibility of the operator

$$
\mathbf{R}^{\mathrm{var}}(G)=\Pi_{[0, \infty)}+\mathbf{M}_{G} \Pi_{(-\infty, 0)}
$$

with $G$ given by (10.1). To this end, consider the equation $\mathbf{R}^{\mathrm{var}}(G) \phi=g$. Using the notation $\phi=\left[\begin{array}{l}\phi_{1} \\ \phi_{2}\end{array}\right], g=\left[\begin{array}{l}g_{1} \\ g_{2}\end{array}\right]\left(\phi_{j}, g_{j} \in(B)^{n \times 1} ; j=1,2\right)$, we rewrite this 
equation in a form of the system

$$
\begin{aligned}
\phi_{1}^{+}+f \phi_{1}^{-}+e^{-i \mu t} \phi_{2}^{-} & =g_{1}, \\
\phi_{2}^{+}+e^{i \mu t} \phi_{1}^{-} & =g_{2},
\end{aligned}
$$

where $\phi_{j}^{+}=\Pi_{[0, \infty)} \phi_{j}, \phi_{j}^{-}=\Pi_{(-\infty, 0)} \phi_{j}(j=1,2)$.

Consider the homogeneous $\left(g_{1}=g_{2}=0\right)$ case first. Equation (10.3) then implies

$$
\phi_{2}^{+}=-e^{i \mu t} \phi_{1}^{-},
$$

so that, in particular,

$$
\sigma\left(\phi_{2}^{+}\right) \subseteq[0, \mu) .
$$

In its turn, the homogeneous equation (10.2) after multiplying by $e^{i \mu t}$ can be rewritten as

$$
e^{i \mu t} \phi_{1}^{+}+f e^{i \mu t} \phi_{1}^{-}+\phi_{2}^{-}=0
$$

or, taking (10.4) into consideration,

$$
e^{i \mu t} \phi_{1}^{+}-f \phi_{2}^{+}+\phi_{2}^{-}=0 \text {. }
$$

Since

$$
\sigma\left(e^{i \mu t} \phi_{1}^{+}\right) \subseteq[\mu, \infty), \sigma\left(\phi_{2}^{-}\right) \subseteq(-\infty, 0),
$$

application of $\Pi_{[0, \mu)}$ to the both sides of (10.6) yields $\Pi_{[0, \mu)} f \phi_{2}^{+}=0$. Due to (10.5), the latter condition means simply that $\widetilde{\mathbf{T}}(f)_{[0, \mu)} \phi_{2}^{+}=0$. From this and the invertibility of $\widetilde{\mathbf{T}}(f)_{[0, \mu)}$ it follows that $\phi_{2}^{+}=0$. According to (10.4), $\phi_{1}^{-}=0$ as well.

Condition (10.6) takes the form

$$
e^{i \mu t} \phi_{1}^{+}+\phi_{2}^{-}=0,
$$

which (because of (10.7)) is possible only if $\phi_{1}^{+}=0$ and $\phi_{2}^{-}=0$. We have proved that the only solution to $\mathbf{R}^{\operatorname{var}}(G) \phi=0$ is $\phi=0$; in other words, that the operator $\mathbf{R}^{\operatorname{var}}(G)$ is injective.

To prove its surjectivity, for arbitrary $g_{1}, g_{2} \in(B)^{n \times 1}$ let

$$
\begin{gathered}
\phi_{1}^{-}=e^{-i \mu t}\left(\Pi_{(-\infty, 0)} g_{2}+\chi\right), \phi_{2}^{+}=\Pi_{[0, \infty)} g_{2}-\chi, \\
\phi_{1}^{+}=\Pi_{[0, \infty)}\left(g_{1}-f \phi_{1}^{-}\right), \phi_{2}^{-}=\Pi_{(-\infty, 0)} e^{i \mu t}\left(g_{1}-f \phi_{1}^{-}\right),
\end{gathered}
$$

where

$$
\chi=\left(\widetilde{\mathbf{T}}(f)_{[0, \mu)}\right)^{-1} \Pi_{[0, \mu)}\left(e^{i \mu t} g_{1}-f \Pi_{(-\infty, 0)} g_{2}\right) .
$$

Obviously, $\phi_{j}^{+} \in(B)_{[0, \infty)}^{n \times 1}$ and $\phi_{j}^{-} \in(B)_{(-\infty, 0)}^{n \times 1}(j=1,2)$. On top of that, with such a choice of $\phi_{j}^{ \pm}$the left hand side of (10.3) equals

$$
\Pi_{[0, \infty)} g_{2}-\chi+\Pi_{(-\infty, 0)} g_{2}+\chi=g_{2} .
$$

Finally, (10.2) can be rewritten as

$$
\phi_{1}^{+}+e^{-i \mu t} \phi_{2}^{-}=g_{1}-f \phi_{1}^{-},
$$

or, substituting $\phi_{1}^{+}$and $\phi_{2}^{-}$,

$$
\Pi_{[0, \infty)}\left(g_{1}-f \phi_{1}^{-}\right)+e^{-i \mu t} \Pi_{(-\infty, 0)} e^{i \mu t}\left(g_{1}-f \phi_{1}^{-}\right)=g_{1}-f \phi_{1}^{-} .
$$


Since $e^{-i \mu t} \Pi_{(-\infty, 0)} e^{i \mu t}=\Pi_{(-\infty,-\mu)}$, equality (10.8) holds if and only if

$$
\Pi_{[0, \mu)}\left(e^{i \mu t} f \phi_{1}^{-}\right)=\Pi_{[0, \mu)}\left(e^{i \mu t} g_{1}\right),
$$

or, using the definition of $\phi_{1}^{-}$,

$$
\Pi_{[0, \mu)}(f \chi)=\Pi_{[0, \mu)}\left(e^{i \mu t} g_{1}-f \Pi_{(-\infty, 0]} g_{2}\right) .
$$

The latter equality holds due to the definition of $\chi$. Hence, the operator $\mathbf{R}^{\mathrm{var}}(G)$ is not only injective but also surjective, and therefore invertible.

For N II the point-excluding analogue of Theorem 4.1 now reads as follows.

Theorem 10.2. Let $f \in(A P W)_{(-\infty, 0) \cap \Sigma}^{m \times n}$ be given, where $\Sigma \subseteq \mathbb{R}$ is an additive subgroup. The following statements are equivalent:

(i) $f$ has a strictly contractive extension in $(A P W)^{m \times n}$, i.e., there exists $g \in$ $(A P W)^{m \times n}$ such that $g_{\lambda}=f_{\lambda}$ for all $\lambda<0$ and $\sup _{t \in \mathbb{R}}\|g(t)\|<1$;

(ii) the generalized Hankel operator

$$
\widetilde{\mathbf{H}}(f):(B)_{[0, \infty)}^{n \times 1} \rightarrow(B)_{(-\infty, 0)}^{m \times 1}, \quad g \mapsto \Pi_{(-\infty, 0)}(f g),
$$

is a strict contraction.

(iii) the generalized Hankel operator

$$
\widetilde{\widetilde{\mathbf{H}}}(f):(B)_{(0, \infty)}^{n \times 1} \rightarrow(B)_{(-\infty, 0]}^{m \times 1}, \quad g \mapsto \Pi_{(-\infty, 0]}(f g),
$$

is a strict contraction.

(iv) $f$ has a strictly contractive extension in $(A P W)_{\Sigma}^{m \times n}$, i.e., there exists $g \in$ $(A P W)_{\Sigma}^{m \times n}$ such that $g_{\lambda}=f_{\lambda}$ for all $\lambda<0$ and $\sup _{g \in \mathbb{R}}\|g(t)\|<1$;

(v) the generalized Hankel operator

$$
\widetilde{\mathbf{H}}(f)_{\Sigma}:(B)_{[0, \infty) \cap \Sigma}^{n \times 1} \rightarrow(B)_{(-\infty, 0) \cap \Sigma}^{m \times 1}, \quad g \mapsto \Pi_{(-\infty, 0)}(f g),
$$

is a strict contraction.

(vi) the generalized Hankel operator

$$
\widetilde{\widetilde{\mathbf{H}}}(f)_{\Sigma}:(B)_{\substack{(0, \infty) \cap \Sigma \\ n \times 1}} \rightarrow(B)_{(-\infty, 0] \cap \Sigma}^{m \times 1}, \quad g \mapsto \Pi_{(-\infty, 0]}(f g),
$$

is a strict contraction.

(vii) the Hankel operator $\hat{\mathbf{H}}(f):\left(H^{2+}\right)^{n \times 1} \rightarrow\left(H^{2-}\right)^{m \times 1}$ is a strict contraction.

When one (and thus all) of (i)-(vii) is satisfied, then put

$$
\begin{aligned}
\widehat{\alpha}(t) & =\left[I-\widetilde{\widetilde{\mathbf{H}}}(f)_{\Sigma}\left(\widetilde{\widetilde{\mathbf{H}}}(f)_{\Sigma}\right)^{*}\right]^{-1}\left(I_{m}\right), \\
\widehat{\beta}(t) & =\widetilde{\mathbf{H}}(f)_{\Sigma}\left[I-\left(\widetilde{\mathbf{H}}(f)_{\Sigma}\right)^{*} \widetilde{\mathbf{H}}(f)_{\Sigma}\right]^{-1}\left(I_{n}\right), \\
\widehat{\gamma}(t) & =\left(\widetilde{\widetilde{\mathbf{H}}}(f)_{\Sigma}\right)^{*}\left[I-\left(\widetilde{\widetilde{\mathbf{H}}}(f)_{\Sigma}\left(\widetilde{\mathbf{H}}(f)_{\Sigma}\right)^{*}\right]^{-1}\left(I_{m}\right),\right. \\
\widehat{\delta}(t) & \left.=\left[I-\left(\widetilde{\mathbf{H}}(f)_{\Sigma}\right)^{*} \widetilde{\mathbf{H}}(f)_{\Sigma}\right)\right]^{-1}\left(I_{n}\right),
\end{aligned}
$$


where $I_{k}$ stands for the constant matrix function on $\mathbb{R}$ with value $I_{k}$ for all $t \in$ $\mathbb{R}$, and the Hankel operators are defined on matrix valued functions columnwise. Further, let

$$
\begin{aligned}
& \alpha(t)=\widehat{\alpha}(t) M\{\widehat{\alpha}\}^{-\frac{1}{2}}, \beta(t)=\widehat{\beta}(t) M\{\widehat{\delta}\}^{-\frac{1}{2}} \\
& \gamma(t)=\widehat{\gamma}(t) M\{\widehat{\alpha}\}^{-\frac{1}{2}}, \delta(t)=\widehat{\delta}(t) M\{\widehat{\delta}\}^{-\frac{1}{2}}
\end{aligned}
$$

Then the function

$$
g_{0}(t)=\beta(t) \delta(t)^{-1}=\left[\alpha(t)^{*}\right]^{-1} \gamma(t)^{*}, t \in \mathbb{R},
$$

is a strictly contractive extension in $(A P W)_{\Sigma}^{m \times n}$ of $f$.

In order to prove Theorem 10.2 one needs to make the following changes for the proof of Theorem 4.1.

(A) Use formula (5.7) in place of (5.6).

(B) Use Remark 9.3 to prove that in a point-excluding analog of Lemma 9.2 the matrix function $G=\left[\begin{array}{cc}I-f f^{*} & f \\ f^{*} & -I\end{array}\right]$ still allows a $W$-canonical factorization of the form (9.7).

(C) Replace the definitions of $y_{+}$and $y_{-}$in (9.13) by

$$
y_{+}=-\gamma \gamma_{0}^{*}+\delta \delta_{0}^{*}, \quad y_{-}=p^{*} \gamma_{0}^{*}+r^{*} \delta_{0}^{*} .
$$

This will result in $M\left\{y_{-}\right\}=0$.

(D) In (9.15) and further, $\mathbf{H}(f)$ needs to be replaced by $\widetilde{\mathbf{H}}(f)$. The $x_{22}^{+}$thus obtained is then the subject of an analog of Lemma 9.4.

(E) In an analog of Lemma 9.5 the operator $\mathbf{H}(f)$ gets replaced by $\widetilde{\mathbf{H}}(f)$.

(F) In the definitions of $\mathcal{M}_{1}$ and $\mathcal{M}_{2}^{0}$ before (9.17) one needs to choose now

$$
g \in(A P W)_{[0, \infty) \cap \Sigma}^{m \times n}, \quad g_{3} \in(A P W)_{(-\infty, 0) \cap \Sigma} .
$$

(G) The equivalence of (vii) with (v) and (vi) follows from Theorem 5.2.

In several other places small changes need to be made (replace $\mathbf{H}(f)$ by $\widetilde{\mathbf{H}}(f)$, replace $(-\infty, 0]$ by $(-\infty, 0)$, etc.) These are now straightforward.

To obtain additional valid results for the point-excluding version of the Nehari problem the following changes in Theorems 4.2, 4.3 and 9.7 need to be made.

(1) Replace $(A P W)_{(-\infty, 0] \cap \Sigma}$ by $(A P W)_{(-\infty, 0) \cap \Sigma}$.

(2) Replace the reference to Theorem 4.1 by a reference to Theorem 10.2 .

(3) Replace $\mathbf{H}(f)_{\Sigma}$ by $\widetilde{\mathbf{H}}(f)_{\Sigma}$ (and omit other Hankel conditions).

(4) Replace $(C A P W)_{(0, \infty) \cap \Sigma}^{m \times n}$ by $(C A P W)_{[0, \infty) \cap \Sigma}^{m \times n}$ in Theorem 4.3.

The proofs require straightforward modifications.

\section{A JOINT NORM BOUND}

Let $\mathbb{T}=\{z:|z|=1\}$ denote the unit circle. In order to prove a $C^{*}$-algebra result it was shown in [25] that for any given $f$ in $L^{\infty}(\mathbb{T})$ and $\epsilon>1$, there exists a function $h$ in $H^{\infty}(\mathbb{T})$ satisfying

$$
\|f+h\|_{\infty} \leq \epsilon d_{\infty} \text { and }\|f+h\|_{2} \leq \frac{\epsilon d_{2}}{\sqrt{\epsilon^{2}-1}}
$$


where $d_{\infty}$ is the distance from $f$ to $H^{\infty}(\mathbb{T})$ in the $L^{\infty}(\mathbb{T})$ norm, and $d_{2}$ is the distance from $f$ to $H^{2}(\mathbb{T})$ in the $L^{2}(\mathbb{T})$ norm. The above inequalities can be understood in the sense of simultaneous $H^{\infty} / H^{2}$ approximation, or optimization (if $\epsilon$ is selected to make a combination of $\|f+h\|_{\infty}$ and $\|f+h\|_{2}$ as small as possible with respect to a specified criterion). Both $H^{\infty}$ and $H^{2}$ optimizations play an essential role in control theory as widely used criteria for control optimization, and recently the simultaneous $H^{\infty} / H^{2}$ optimization has been recognized as an important tool in the multiobjective control (see, e.g., $[8,3,36]$ ).

In this section we present an almost periodic version of this result. The proof uses ideas of the proof of the result in [25] as presented in [15] (see also the appendix in [14]).

For $f \in(B)^{m \times n}$ let $\|f\|_{B}=\left[\operatorname{tr}\left(M\left\{f^{*} f\right\}\right)\right]^{1 / 2}$ be the Besicovitch norm.

Theorem 11.1. Let $\Sigma$ be an additive subgroup of $\mathbb{R}$, and let $f \in(A P W)_{(-\infty, 0] \cap \Sigma}^{m \times n}$ be given. Denote $\widehat{d}_{2}=\|f\|_{B}, \widehat{d}_{\infty}=\|\mathbf{H}(f)\|$. For each $\epsilon>1$ there exists an $\tilde{f} \in(A P W)_{(0, \infty) \cap \Sigma}^{m \times n}$ such that

$$
\|f+\widetilde{f}\| \leq \epsilon \widehat{d}_{\infty} \text { and }\|f+\widetilde{f}\|_{B} \leq \frac{\epsilon \widehat{d}_{2}}{\sqrt{\epsilon^{2}-1}}
$$

Proof. Without loss of generality we may assume that $\epsilon \widehat{d}_{\infty}=1$ (excluding the trivial case $f \equiv 0)$, since we may divide $f$ by $\epsilon \widehat{d}_{\infty}$. But then

$$
\|\mathbf{H}(f)\|=\frac{1}{\epsilon}<1 .
$$

Now let

$$
\widetilde{f}(t)=\beta(t) \delta(t)^{-1}-f(t),
$$

where $\beta$ and $\delta$ are as in Theorem 4.1. For a complex number $z$ with $|z|<1$ we have

$$
|z|^{2} \leq-\log \left(1-|z|^{2}\right)
$$

and consequently for $M \in \mathbb{C}^{m \times n}$ with $\|M\|<1$ we have

$$
\operatorname{tr} M^{*} M \leq-\log \operatorname{det}\left(I-M^{*} M\right) .
$$

But then it follows that

$$
\begin{aligned}
\|f+\widetilde{f}\|_{B}^{2} & =\lim _{T \rightarrow \infty} \frac{1}{2 T} \int_{-T}^{T} \operatorname{tr}\left[((f+\widetilde{f})(t))^{*}(f+\widetilde{f})(t)\right] d t \\
& \leq-\lim _{T \rightarrow \infty} \frac{1}{2 T} \int_{-T}^{T} \log \operatorname{det}\left(I-((f+\widetilde{f})(t))^{*}(f+\widetilde{f})(t)\right) d t \\
& =-\lim _{T \rightarrow \infty} \frac{1}{2 T} \int_{-T}^{T} \log \operatorname{det}\left(I-\left(\beta(t) \delta(t)^{-1}\right)^{*} \beta(t) \delta(t)^{-1}\right) d t .
\end{aligned}
$$

By the proof of Theorem 4.3 (use the last equality with $g=0$ ) we have that

$$
\begin{aligned}
I-\left(\beta(t) \delta(t)^{-1}\right)^{*} \beta(t) \delta(t)^{-1} & =\delta(t)^{-1 *}\left(\delta(t)^{*} \delta(t)-\beta(t)^{*} \beta(t)\right) \delta(t)^{-1} \\
& =\delta(t)^{-1 *} \delta(t)^{-1} .
\end{aligned}
$$

Since $\left(\delta(t) M\{\delta\}^{-1}\right)^{ \pm 1} \in I+(A P W)_{(0, \infty)}^{n \times n}$, we get from Proposition 2.9 that

$$
M\left\{\log \left(\delta(t) M\{\delta\}^{-1}\right)\right\}=0 .
$$


So then we obtain

$$
\begin{gathered}
\|f+\widetilde{f}\|_{B}^{2} \leq M\left\{\log \operatorname{det}\left(\delta(t)^{-1 *} \delta(t)^{-1}\right)\right\} \\
=\log \operatorname{det}(M\{\widehat{\delta}\})=\operatorname{tr} \log M\{\widehat{\delta}\},
\end{gathered}
$$

where $\widehat{\delta}$ is as in Theorem 4.1. Note that

$$
\begin{aligned}
M\{\hat{\delta}\} & =M\left\{\left(I-(\mathbf{H}(f))^{*} \mathbf{H}(f)\right)^{-1}\left(I_{n}\right)\right\} \\
& \left.=M\left\{\left(I+(\mathbf{H}(f))^{*}(I-\mathbf{H}(f)(\mathbf{H})(f))^{*}\right)^{-1} \mathbf{H}(f)\right)\left(I_{n}\right)\right\} .
\end{aligned}
$$

From the inequality $\log (1+r) \leq r$, valid for $r \geq 0$, we get

$$
\log M\{\widehat{\delta}\} \leq M\left\{\left(\mathbf{H}(f)^{*}\left(I-\mathbf{H}(f) \mathbf{H}(f)^{*}\right)^{-1} \mathbf{H}(f)\right)\left(I_{n}\right)\right\} .
$$

Since $\|\mathbf{H}(f)\| \leq \widehat{d}_{\infty}=\frac{1}{\epsilon}$, from this and from (11.1) it follows that

$$
\|f+\widetilde{f}\|_{B}^{2} \leq \frac{1}{1-\widehat{d}_{\infty}^{2}} \operatorname{tr} M\left\{\left(\mathbf{H}(f)^{*} \mathbf{H}(f)\right)\left(I_{n}\right)\right\}=\frac{\epsilon^{2}}{\epsilon^{2}-1}\|f\|_{B}^{2}=\frac{\epsilon^{2} \widehat{d}_{2}^{2}}{\epsilon^{2}-1} .
$$

By using Theorem 10.2 instead of Theorem 4.1 one can prove a point-excluding variation of Theorem 11.1 where $f \in(A P W)_{(-\infty, 0) \cap \Sigma}^{m \times n}, \widetilde{f} \in(A P W)_{[0, \infty) \cap \Sigma}^{m \times n}$, and $\tilde{d}_{\infty}=\|\tilde{\mathbf{H}}(f)\|$.

\section{REFERENCES}

[1] V. M. Adamjan, D. Z. Arov, and M. G. Krein. Infinite Hankel block matrices and related extension problem. Izv. Akad. Nauk SSSR. Ser. Mat., 6:87-112, 1971. English Transl.: Amer. Math. Soc. Trans. (2) 111: 133-156, 1978. MR 45:7506

[2] J. A. Ball, I. Gohberg, and L. Rodman. Interpolation of Rational Matrix Functions. OT45. Birkhäuser, 1990. MR 92m:47027

[3] D. S. Bernstein and W. M. Haddad. $L Q C$ control with an $H_{\infty}$ performance bound: a Riccati equation approach. IEEE Trans. Autom. Control, AC-34:293-305, 1989. MR 90h: 49025

[4] J. P. Burg. Maximum Entropy Spectral Analysis. PhD thesis, Department of Geophysics, Stanford University, Stanford, California, 1975.

[5] C. Carathéodory. Über den Variabilitätsbereich der Fourierschen Konstanten von positiven harmonischen Funktionen. Rendiconti dei Circolo Matematico di Palermo, 32:193$217,1911$.

[6] C. Corduneanu. Almost Periodic Functions. J. Wiley \& Sons, 1968. MR 58:2006

[7] A. Devinatz and M. Shinbrot. General Wiener-Hopf operators. Trans. Amer. Math. Soc., 145:467-494, 1969. MR 40:4800

[8] J. C. Doyle, K. Glover, P. Khargonekar, and B. A. Francis. State-space solutions to standard $\mathrm{H}_{2}$ and $\mathrm{H}_{\infty}$ control problems. IEEE Trans. on Automatic Control, 34(8):831847, 1989. MR 90m:93055

[9] H. Dym and I. Gohberg. Extensions of matrix valued functions with rational polynomial inverse. Integral Equations and Operator Theory, 2:503-528, 1979. MR 81g:47015

[10] H. Dym and I. Gohberg. Extensions of kernels of Fredholm operators. J. Anal. Math, 42:51-97, 1982/83. MR 86b:45002

[11] H. Dym and I. Gohberg. A maximum entropy principle for contractive interpolants. J. Functional Analysis, 65:83-125, 1986. MR 87h:47065

[12] H. Dym and I. Gohberg. A new class of contractive interpolants and maximum entropy principles. Operator Theory: Advances and Applications, 29:117-150, 1988. MR 89h:47022

[13] C. Foias and A. E. Frazho. The Commutant Lifting Approach to Interpolation Problems. OT44. Birkhäuser, 1990. MR 92k:47033 
[14] A. E. Frazho and S. M. Kherat. Applications of the Schur algorithm to mixed $H^{2}$ and $H^{\infty}$ Nehari problems. J. Math. Anal. Appl., 178(2):488-508, 1993. MR 94h:47026

[15] A. E. Frazho and M. A. Rotea. A remark on mixed $L^{2} / L^{\infty}$ bounds. Integral Equations and Operator Theory, 15(2):343-348, 1992. MR 93b:47029

[16] I. Gelfand, D. Raikov, and G. Shilov. Commutative Normed Rings. Chelsea, Bronx, N.Y., 1964. MR 34:4940

[17] I. Gohberg, S. Goldberg, and M. A. Kaashoek. Classes of Linear Operators, II. OT63. Birkhäuser, 1993. MR 95a:47001

[18] I. Gohberg, M. A. Kaashoek, and H. J. Woerdeman. The band method for positive and contractive extension problems. J. Operator Theory, 22:109-155, 1989. MR 91a:47021

[19] I. Gohberg, M. A. Kaashoek, and H. J. Woerdeman. The band method for positive and contractive extension problems: An alternative version and new applications. Integral Equations and Operator Theory, 12:343-382, 1989. MR 90c: 47022

[20] I. Gohberg, M. A. Kaashoek, and H. J. Woerdeman. The band method for extension problems and maximum entropy. In L. Auslander, T. Kailath, and S. Mitter, editors, Signal Processing; Part I, volume 22 of The IMA Volumes in Mathematics and its Applications, New York, 1990. Springer Verlag, pp. 75-94. MR 91e:47012

[21] I. Gohberg, M. A. Kaashoek, and H. J. Woerdeman. The band method for several positive extension problems of non-band type. J. Operator Theory, 26:191-218, 1991. MR 94e: 47027

[22] I. Gohberg, M. A. Kaashoek, and H. J. Woerdeman. A maximum entropy principle in the general framework of the band method. J. Functional Analysis, 95:231-254, 1991. MR 92b: 47020

[23] S. Goldberg. Unbounded Linear Operators: Theory and Applications. McGraw-Hill, New York, 1966. MR 34:580

[24] J. W. Helton. Operator Theory, Analytic Functions, Matrices, and Electrical Engineering, volume 68 of CBMS Conference Series. American Mathematical Society, 1987. MR 89f: 47001

[25] V. G. Kaftal, D. R. Larson, and G. Weiss. Quasitriangular subalgebras of semifinite von Neumann subalgebras are closed. J. Functional Analysis, 107:387-401, 1992. MR 93g:46060

[26] Yu. I. Karlovich. The local trajectory method of studying invertibility in $C^{*}$-algebras of operators with discrete groups of shifts. Soviet Math. Dokl., 37:407-412, 1988. MR 89e: 46068

[27] Yu. I. Karlovich. Algebras of convolution type operators with discrete groups of shifts and oscillating coefficients. Doctoral dissertation, Mathematical Institute, Georgian Academy of Sciences, Tbilisi, 1991. (Russian)

[28] Yu. I. Karlovich and G. S. Litvinchuk. On some classes of semi-Fredholm operators. Izvestiya VUZov. Mat., (2):3-16, 1990; English transl. in Soviet Math. (Iz. VUZ). MR 91k:47122

[29] Yu. I. Karlovich and I. M. Spitkovsky. Semi-Fredholm properties of certain singular integral operators. In Operator Theory: Advances and Applications, vol. 90, Birkhäuser, 1996, pp. 269-287. CMP 97:02

[30] Yu. I. Karlovich and I. M. Spitkovsky. (Semi)-Fredholmness of convolution operators on the spaces of Bessel potentials. Operator Theory: Advances and Applications, 71:122152, 1994. MR 95h:47034

[31] B. M. Levitan. Almost Periodic Functions. GITTL, Moscow, 1953. (Russian) MR 15:700a

[32] B. M. Levitan and V. V. Zhikov. Almost Periodic Functions and Differential Equations. Cambridge University Press, 1982. MR 84g:34004

[33] M. Morf, A. Vieira, and T. Kailath. Covariance characterization by partial autocorrelation matrices. Ann. Statist., 6:643-648, 1978. MR 57:17996

[34] M. Morf, A. Vieira, D. Lee, and T. Kailath. Recursive multichannel maximal entropy spectral estimation. IEEE Trans. Geosci. Elect., 16:85-94, 1978. MR 80e:62066

[35] Z. Nehari. On bounded bilinear forms. Ann. of Math., 104:570-578, 1957. MR 18:633f

[36] M. A. Rotea and P. P. Khargonekar. $H_{2}$-optimal control with an $H_{\infty}$-constraint, the state-feedback case. IFAC Automatica, 27:307-316, 1991. MR 92d:93058 
[37] I. M. Spitkovsky. On the factorization of almost periodic matrix functions. Math. Notes, 45(5-6):482-488, 1989. MR 90k:47033

[38] I. M. Spitkovsky and H. J. Woerdeman. The Carathéodory-Toeplitz problem for almost periodic functions. J. Functional Analysis, 115(2):281-293, 1993. MR 94f:47020

[39] O. Toeplitz. Über die Fourier'sche Entwicklung positiven Funktionen. Rendiconti dei Circolo Matematico di Palermo, 32:1-2, 1911.

[40] H. J. Woerdeman. Matrix and Operator Extensions. CWI Tract 68. Centre for Mathematics and Computer Science, Amsterdam, the Netherlands, 1989. MR 91d:47001

Department of Mathematics, College of William and Mary, Williamsburg, Virginia $23187-8795$

E-mail address: lxrodm@math.wm.edu

E-mail address: ilya@math.wm.edu

E-mail address: hugo@math.wm.edu 